\title{
Digital Compensation of Frequency-Dependent Joint Tx/Rx I/Q Imbalance in OFDM Systems Under High Mobility
}

\author{
Balachander Narasimhan, Student Member, IEEE, Dandan Wang, Student Member, IEEE, \\ Sudharsan Narayanan, Student Member, IEEE, Hlaing Minn, Senior Member, IEEE, and \\ Naofal Al-Dhahir, Fellow, IEEE
}

\begin{abstract}
Direct conversion orthogonal frequency division multiplexing (OFDM) systems suffer from transmit and receive analog processing impairments such as in-phase/quadrature (I/Q) imbalance causing inter-carrier interference (ICI) among sub-carriers. Another source of performance-limiting ICI in OFDM systems is Doppler spread due to mobility. However, the nature of ICI due to each of them is quite different. Unlike previous work which considered these two impairments separately, we develop a unified mathematical framework to characterize, estimate, and jointly mitigate ICI due to I/Q imbalance and high mobility. Based on our general model, we derive a closed-form expression for the degradation in signal-to-interference-plus-noise ratio (SINR) due to the impairments. Moreover, we exploit the special ICI structure to design efficient OFDM channel estimation and digital baseband compensation schemes for joint transmit/receive frequency-independent (FI) and frequency-dependent (FD) I/Q imbalances under highmobility conditions.
\end{abstract}

Index Terms-Channel estimation, in-phase/quadrature (I/Q) imbalance, inter-carrier interference (ICI), minimum mean square error (MMSE) equalization, mobility, orthogonal frequency division multiplexing (OFDM), signal-to-interference-plus-noise ratio (SINR).

\section{INTRODUCTION}

$\mathbf{N}$ EXT-GENERATION broadband wireless systems are required to provide higher rates, better reliability, and higher user mobility while targeting lower cost, lower power consumption, and higher levels of integration (smaller form factor). Orthogonal frequency division multiplexing (OFDM) has been adopted as the transmission technology for most broadband wireless standards (such as IEEE 802.16 and IEEE 802.11). To support higher information rates, the future trend is to operate at higher carrier frequencies and use higher-order signal constellations (e.g., 64 QAM) which are more sensitive to mobility and to implementation imperfections such as I/Q imbalance.

Manuscript received June 01, 2008; revised February 10, 2009. Current version published May 15, 2009. This work was supported by gifts from Texas Instruments, Inc., and Research in Motion, Inc. The associate editor coordinating the review of this manuscript and approving it for publication was Prof. Ali Sayed.

The authors are with the Department of Electrical Engineering, University of Texas at Dallas, Richardson, TX 75080 USA (e-mail: bxn062000@utdallas.edu; dxw053000@utdallas.edu; sxn062100@utdallas.edu; hlaing.minn@utdallas.edu; aldhahir@utdallas.edu).

Digital Object Identifier 10.1109/JSTSP.2009.2020325
I/Q Imbalance refers to amplitude and phase mismatches between the in-phase (I) and quadrature (Q) branches of a transceiver. Ideally, the I and Q branches of the mixers should have equal amplitude and $90^{\circ}$ phase shift but this is rarely the case in practice, resulting in FI I/Q imbalance. However, those occurring in other analog components such as transmit/receive analog filters, amplifiers, and D/A or A/D converters, could be FD. $\mathrm{I} / \mathrm{Q}$ imbalance is especially pronounced in direct-conversion receivers and like other impairments in the analog components, it is exacerbated due to fabrication process variations which are difficult to predict or control, increase with the down-scaling of fabrication technologies, and cannot be efficiently or completely canceled in the analog domain due to power-area-cost tradeoffs.

Besides I/Q imbalance, mobility (Doppler effect) also destroys sub-carrier orthogonality within each OFDM symbol by introducing inter-carrier interference (ICI) which becomes more severe at higher speeds, higher carrier frequencies, and for larger OFDM block durations (necessary to combat severe channel frequency selectivity). All of the above-mentioned considerations coupled with the fact that embedded digital processors and custom ASICs in mobile devices are becoming more powerful, motivate this research which aims at developing high-performance low-complexity digital baseband compensation techniques for I/Q imbalance in mobile OFDM systems.

Digital compensation of I/Q imbalance in OFDM systems has been investigated in several recent papers. A representative (but not comprehensive) list is [1]-[8]. This paper is distinct from previous research in this area in the following major contribution. Previous work in this area considers either I/Q imbalance or mobility. However, in broadband outdoor wireless systems (such as WiMAX, 3GPP LTE or DVB-H), it is likely that both impairments will be present. We develop a generalized mathematical model to quantify and compensate for the joint ICI effects of both impairments. This will allow us to evaluate the individual as well as the combined effects of these impairments on system performance, better understand their interactions, and identify the dominant impairment(s) under a specific operating scenario. Towards this end, a closed-form signal-tointerference-plus-noise ratio (SINR) expression is derived to quantify the effects of both I/Q imbalance and mobility. Furthermore, we exploit the channel and ICI structure to reduce the complexity of digital baseband compensation algorithms. Finally, effective compensation of I/Q imbalance and mobility and reliable coherent signal detection require accurate channel estimates at the receiver in the presence of these impairments. This 
is a very challenging task especially for broadband channels under high mobility (due to the increased number of channel parameters to be estimated and due to fast channel time variations). Based on our derived model, we first propose a general channel estimation scheme for both FI and FD I/Q imbalances. Then, a new channel estimation scheme based on I/Q imbalance parameter ratios is proposed for FI I/Q imbalance to reduce the pilot overhead.

This paper is organized as follows. In Section II, we develop a general input-output model in the presence of joint transmit/ receive FD and FI I/Q imbalances in OFDM systems under high mobility. In Section III, we derive the SINR performance degradation in the presence of the impairments. A reduced-complexity digital baseband joint compensation scheme is presented in Section IV. In Section V, two channel estimation schemes are proposed. Simulation results are given in Section VI and the paper is concluded in Section VII.

Notation: Functions $x(\cdot)$ are denoted by lowercase letters. All time-domain quantities $\{\bar{x}(t), \overline{\mathbf{x}}, \overline{\mathbf{X}}\}$ have a bar whereas frequency-domain quantities $\{x(f), \mathbf{x}, \mathbf{X}\}$ do not. Vectors $\{\overline{\mathbf{x}}, \mathbf{x}\}$ are represented by lowercase boldface. Matrices $\{\overline{\mathbf{X}}, \mathbf{X}\}$ are represented by uppercase boldface letters. $a_{k}$ represents the $k$ th element of a and $a_{k, l}$ represents the $(k, l)$-element of matrix $\mathbf{A}$. Conjugate, transpose and Hermitian operations are denoted by $(\cdot)^{*},(\cdot)^{T}$ and $(\cdot)^{H}$, respectively. $N$ is the size of the discrete Fourier transform (DFT). F is the unitary DFT matrix whose $(n, k)$ element is $(1 / \sqrt{N}) e^{-j(2 \pi) /(N) k n}$ for $0 \leq n, k \leq N-1$. Linear and circular (modulo- $N$ ) convolutions are denoted by $\otimes$ and $\circledast_{N}$, respectively. $\mathbf{I}_{k}$ is the identity matrix of size $k$. $\mathbf{0}_{m \times n}$ represents the all-zero matrix of size $m \times n$. The operator $\operatorname{Diag}(\cdot)$, when applied to a matrix results in a vector containing the diagonal elements of the matrix and when it acts on a vector results in a diagonal matrix whose diagonal elements are the elements of the vector. All signals are indexed modulo- $N$, i.e., $x(k)=x((N-k))_{N} \cdot \Re\{\cdot\}$ and $\Im\{\cdot\}$ denote the real and imaginary parts of a complex number, respectively. $E\{\cdot\}$ is the expectation operator and $\delta(\cdot)$ is the dirac-delta function.

\section{SySTEM MODEL AND ASSUMPTIONS}

\section{A. General I/Q Imbalance Model}

In this section, we present a general mathematical model for FD joint Tx/Rx I/Q imbalance. The system block diagram under consideration is shown in Fig. 1. Let $\bar{s}(n)=\bar{s}^{I}(n)+j \bar{s}^{Q}(n)$ denote the discrete-time complex baseband signal at the transmitter which passes through the digital-to-analog converter (DAC) and a pulse-shaping or low-pass filter (LPF). We model the combined DAC and filters in the $\mathrm{I}$ and $\mathrm{Q}$ branches as a cascade of the desired LPF denoted as $\bar{h}_{t}(t)$ and a filter representing the mismatch between the I and $\mathrm{Q}$ branches, denoted as $\bar{\xi}_{t}^{I}(t)$ and $\bar{\xi}_{t}^{Q}(t)$, respectively. Note that the Fourier transforms of $\bar{\xi}_{t}^{I}(t)$ and $\bar{\xi}_{t}^{Q}(t)$ could be FD in practical systems and different from each other, which makes the I/Q imbalance FD. Then, the signal components are up-converted using the quadrature tones of the mixer which (ideally) should have equal amplitudes and a phase difference of $90^{\circ}$. However, in most practical systems, this is not the case, which results in FI I/Q imbalance. The amplitude and phase imbalances of the transmit-side mixers are denoted by $a_{t}^{I}, a_{t}^{Q}$ and $\theta_{t}^{I}, \theta_{t}^{Q}$, respectively. The corresponding continuous-time ideal signal generated at the LPF output is $\bar{s}(t)=\bar{s}^{I}(t)+j \bar{s}^{Q}(t)$, where

$$
\begin{aligned}
\bar{s}^{I}(t) & =\Sigma_{n=-\infty}^{\infty} \bar{s}^{I}(n) \bar{h}_{t}(t-n T) \\
\bar{s}^{Q}(t) & =\Sigma_{n=-\infty}^{\infty} \bar{s}^{Q}(n) \bar{h}_{t}(t-n T)
\end{aligned}
$$

$$
\begin{aligned}
& \bar{z}^{I}(t)=\bar{\xi}_{r}^{I}(t) \otimes \bar{r}^{I}(t) \\
& =\left(\frac{\mu_{r} \bar{y}(t)+\nu_{r} \bar{y}^{*}(t)+\mu_{r}^{*} \bar{y}^{*}(t)+\nu_{r}^{*} \bar{y}(t)}{2}\right) \otimes \bar{\xi}_{r}^{I}(t) \otimes \bar{h}_{r}(t) \\
& \bar{z}^{Q}(t)=\bar{\xi}_{r}^{Q}(t) \otimes \bar{r}^{Q}(t) \\
& =\left(\frac{\mu_{r} \bar{y}(t)+\nu_{r} \bar{y}^{*}(t)-\mu_{r}^{*} \bar{y}^{*}(t)-\nu_{r}^{*} \bar{y}(t)}{2 j}\right) \otimes \bar{\xi}_{r}^{Q}(t) \otimes \bar{h}_{r}(t) \\
& \bar{z}(t)=\underbrace{\frac{1}{2}\left(\left(\mu_{r}+\nu_{r}^{*}\right) \bar{\xi}_{r}^{I}(t)+\left(\mu_{r}-\nu_{r}^{*}\right) \bar{\xi}_{r}^{Q}(t)\right)}_{\bar{\lambda}_{r}(t)} \otimes \bar{h}_{r}(t) \otimes \bar{y}(t) \\
& +\underbrace{\frac{1}{2}\left(\left(\nu_{r}+\mu_{r}^{*}\right) \bar{\xi}_{r}^{I}(t)+\left(\nu_{r}-\mu_{r}^{*}\right) \bar{\xi}_{r}^{Q}(t)\right)}_{\bar{\phi}_{r}(t)} \otimes \bar{h}_{r}(t) \otimes \bar{y}^{*}(t) . \\
& \bar{z}(t)=\underbrace{\left[\bar{\lambda}_{r}(t) \otimes \bar{g}(t, \tau) \otimes \bar{\lambda}_{t}(t)+\bar{\phi}_{r}(t) \otimes \bar{g}^{*}(t, \tau) \otimes \bar{\phi}_{t}^{*}(t)\right]}_{\bar{\psi}^{(1)}(t, \tau)} \otimes \bar{h}_{r}(t) \otimes \bar{s}(t) \\
& +\underbrace{\left[\bar{\lambda}_{r}(t) \otimes \bar{g}(t, \tau) \otimes \bar{\phi}_{t}(t)+\bar{\phi}_{r}(t) \otimes \bar{g}^{*}(t, \tau) \otimes \bar{\lambda}_{t}^{*}(t)\right]}_{\bar{\psi}^{(2)}(t, \tau)} \otimes \bar{h}_{r}(t) \otimes \bar{s}^{*}(t) \\
& +\bar{\lambda}_{r}(t) \otimes \bar{h}_{r}(t) \otimes \bar{w}(t)+\bar{\phi}_{r}(t) \otimes \bar{h}_{r}(t) \otimes \bar{w}^{*}(t) .
\end{aligned}
$$




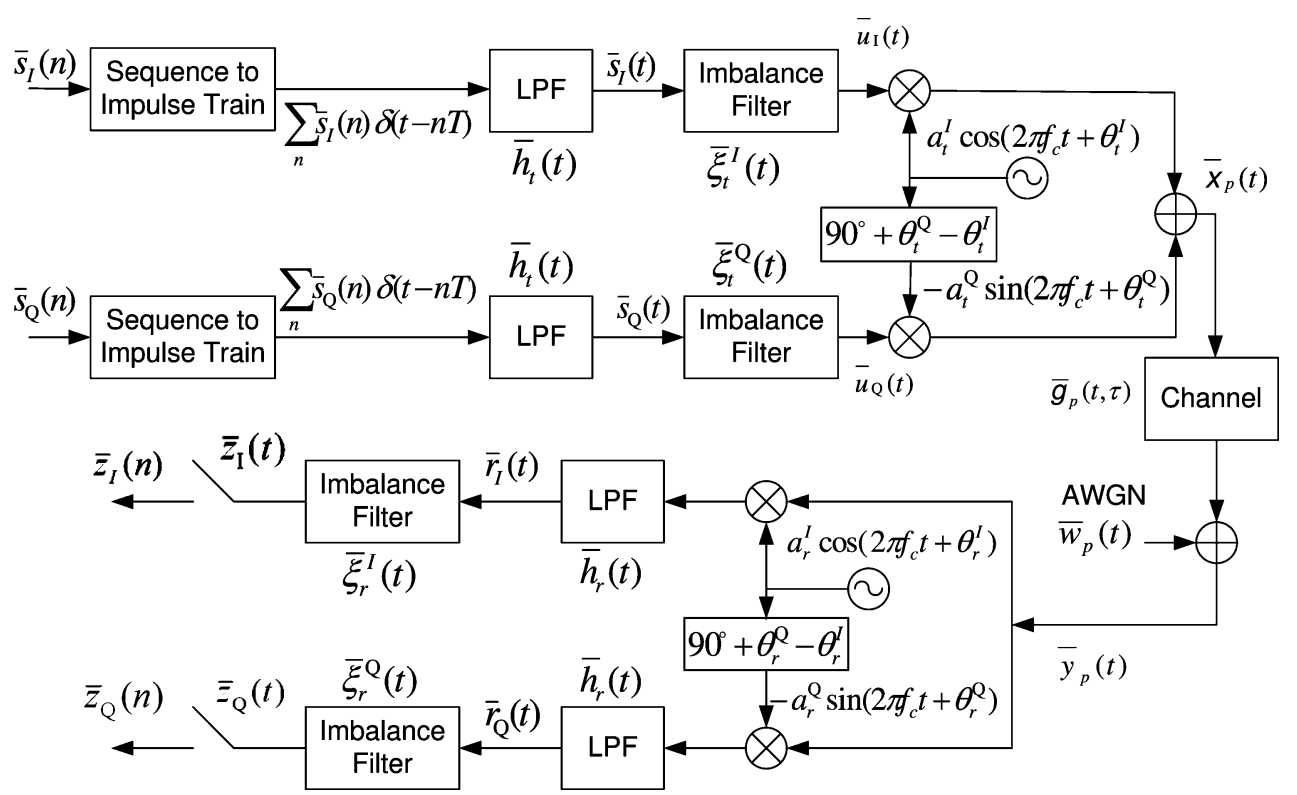

Fig. 1. Block diagram of the joint $\mathrm{I} / \mathrm{Q}$ imbalance and mobility model.

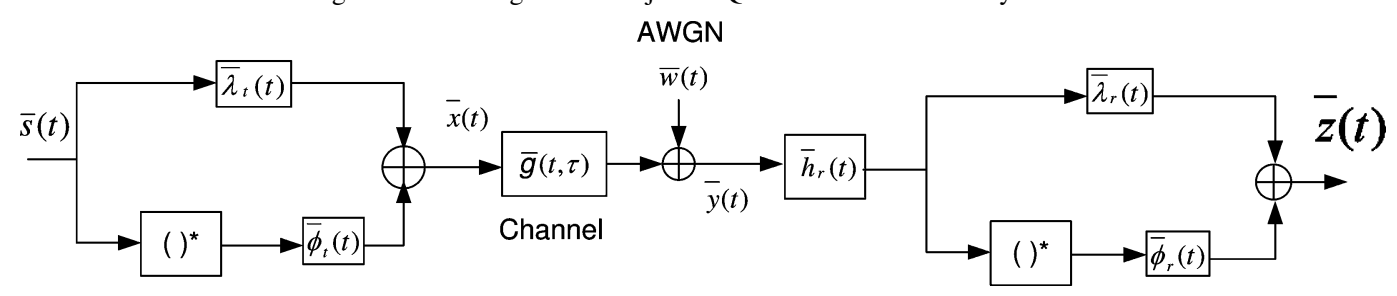

Fig. 2. Block diagram of the equivalent model.

and $T$ is the OFDM sampling duration. Let $\bar{u}(t)=\bar{u}^{I}(t)+$ $j \bar{u}^{Q}(t)$ denote the signal distorted by FD I/Q imbalance before the mixer given by

$$
\bar{u}(t)=\bar{s}^{I}(t) \otimes \bar{\xi}_{t}^{I}(t)+j \bar{s}^{Q}(t) \otimes \bar{\xi}_{t}^{Q}(t)
$$

Also, let $\bar{x}_{p}(t)=\Re\left\{\bar{x}(t) e^{j 2 \pi f_{c} t}\right\}$ and $\bar{x}(t)=\bar{x}^{I}(t)+j \bar{x}^{Q}(t)$ denote, respectively, the passband signal and the baseband equivalent signal after the mixer. When there is no imbalance between the mixer branches, $\bar{x}(t)=\bar{u}(t)$. However, when there is imbalance, we have

$$
\begin{aligned}
\bar{x}_{p}(t)= & \bar{u}^{I}(t) a_{t}^{I} \cos \left(2 \pi f_{c} t+\theta_{t}^{I}\right) \\
& -\bar{u}^{Q}(t) a_{t}^{Q} \sin \left(2 \pi f_{c} t+\theta_{t}^{Q}\right) \\
= & \bar{x}^{I}(t) \cos \left(2 \pi f_{c} t\right)-\bar{x}^{Q}(t) \sin \left(2 \pi f_{c} t\right)
\end{aligned}
$$

which is same as $\Re\left\{\bar{x}(t) e^{j 2 \pi f_{c} t}\right\}$ and

$$
\begin{aligned}
& \bar{x}^{I}(t)=\left(\bar{u}^{I}(t) a_{t}^{I} \cos \theta_{t}^{I}-\bar{u}^{Q}(t) a_{t}^{Q} \sin \theta_{t}^{Q}\right) \\
& \bar{x}^{Q}(t)=\left(\bar{u}^{Q}(t) a_{t}^{Q} \cos \theta_{t}^{Q}+\bar{u}^{I}(t) a_{t}^{I} \sin \theta_{t}^{I}\right)
\end{aligned}
$$

represent the corresponding baseband signal components. Substituting $\bar{u}^{I}(t)=\left(\bar{u}(t)+\bar{u}^{*}(t)\right) /(2)$ and $\bar{u}^{Q}(t)=(\bar{u}(t)-$ $\left.\bar{u}^{*}(t)\right) /(2 j)$ in (6) and grouping the terms carefully, we get

$$
\bar{x}(t)=\mu_{t} \bar{u}(t)+\nu_{t} \bar{u}^{*}(t)
$$

where $\mu_{t}=(1 / 2)\left(a_{t}^{I} e^{j \theta_{t}^{I}}+a_{t}^{Q} e^{j \theta_{t}^{Q}}\right)$ and $\nu_{t}=(1 / 2)\left(a_{t}^{I} e^{j \theta_{t}^{I}}-\right.$ $\left.a_{t}^{Q} e^{j \theta_{t}^{Q}}\right)$. Substituting (4) in (6) and using the relations $\bar{s}^{I}(t)=$ $\left(\bar{s}(t)+\bar{s}^{*}(t)\right) / 2$ and $\bar{s}^{Q}(t)=\left(\bar{s}(t)-\bar{s}^{*}(t)\right) / 2 j$, we obtain

$$
\begin{aligned}
\bar{x}(t)= & \underbrace{\frac{1}{2}\left(\left(\mu_{t}+\nu_{t}\right) \bar{\xi}_{t}^{I}(t)+\left(\mu_{t}-\nu_{t}\right) \bar{\xi}_{t}^{Q}(t)\right)}_{\bar{\lambda}_{t}(t)} \otimes \bar{s}(t) \\
& +\underbrace{\frac{1}{2}\left(\left(\mu_{t}+\nu_{t}\right) \bar{\xi}_{t}^{I}(t)-\left(\mu_{t}-\nu_{t}\right) \bar{\xi}_{t}^{Q}(t)\right)}_{\bar{\phi}_{t}(t)} \otimes \bar{s}^{*}(t) .
\end{aligned}
$$

Then, $\bar{x}_{p}(t)$ is transmitted through the channel and the received passband signal is

$$
\bar{y}_{p}(t)=\bar{g}_{p}(t, \tau) \otimes \bar{x}_{p}(t)+\bar{w}_{p}(t)
$$

which has the baseband equivalent signal

$$
\bar{y}(t)=\bar{g}(t, \tau) \otimes \bar{x}(t)+\bar{w}(t)
$$

where $\bar{g}_{p}(t, \tau)=2 \Re\left\{\bar{g}(t, \tau) e^{j 2 \pi f_{c} t}\right\}$ is the channel impulse response (CIR) and $\bar{w}_{p}(t)=\Re\left\{\bar{w}(t) e^{j 2 \pi f_{c} t}\right\}$ is additive white Gaussian noise (AWGN) with two-sided power spectral density of $\sigma^{2}$. The received signal is down-converted and input to the receive LPF in each branch. The impulse response of the receive LPF $\bar{h}_{r}(t)$ is matched to $\bar{h}_{t}(t)$ and the FD I/Q imbalances are modeled by the cascaded filters $\bar{\xi}_{r}^{I}(t)$ and $\bar{\xi}_{r}^{Q}(t)$. Denote the amplitudes and the phase imbalances of the receiver quadrature mixer tones by $a_{r}^{I}, a_{r}^{Q}$ and $\theta_{r}^{I}, \theta_{r}^{Q}$, respectively. With 
$y_{p}(t)=\Re\left\{\bar{y}(t) e^{j 2 \pi f_{c} t}\right\}=(1 / 2)\left(\bar{y}(t) e^{j 2 \pi f_{c} t}+\bar{y}^{*}(t) e^{-j 2 \pi f_{c} t}\right)$, the output of the ideal receive LPF is

$$
\bar{r}(t)=\frac{1}{2} \bar{h}_{r}(t) \otimes\left(\mu_{r} \bar{y}(t)+\nu_{r} \bar{y}^{*}(t)\right)
$$

where $\mu_{r}=(1 / 2)\left(a_{r}^{I} e^{-j \theta_{r}^{I}}+a_{r}^{Q} e^{-j \theta_{r}^{Q}}\right)$ and $\nu_{r}=$ $(1 / 2)\left(a_{r}^{I} e^{j \theta_{r}^{I}}-a_{r}^{Q} e^{j \theta_{r}^{Q}}\right)$. After experiencing receive-side FD I/Q imbalance, the I and Q components are given by (1), and the final output signal $\bar{z}(t)=\bar{z}^{I}(t)+j \bar{z}^{Q}(t)$ is given by (3). Substituting (8) and (10) into (3), we obtain the relation (3) between $\bar{z}(t)$ and $\bar{s}(t)$. Equation (3) represents an equivalent model that is illustrated in Fig. 2. It is a generalization of the FI I/Q imbalance model in [4], in that $\mu_{t}, \nu_{t}, \mu_{r}$ and $\nu_{r}$ are replaced by $\bar{\lambda}_{t}(t), \bar{\phi}_{t}(t), \bar{\lambda}_{r}(t)$ and $\bar{\phi}_{r}(t)$, respectively.

Note on Symmetric Imbalances: Symmetric imbalance is the case when the amplitude and phase imbalances are distributed equally among the I and Q branches. On the transmit-side, this special case implies that $a_{t}^{I}=1+\tilde{\alpha}_{t}, a_{t}^{Q}=1-\tilde{\alpha}_{t}, \theta_{t}^{I}=-\theta_{t} / 2$, and $\theta_{t}^{Q}=\theta_{t} / 2$. In this case, the expressions for $\mu_{t}$ and $\nu_{t}$ in (7) simplify to

$$
\begin{aligned}
\mu_{t} & =\left(\cos \frac{\theta_{t}}{2}-j \tilde{\alpha}_{t} \sin \frac{\theta_{t}}{2}\right) \\
\nu_{t} & =\left(\tilde{\alpha}_{t} \cos \frac{\theta_{t}}{2}-j \sin \frac{\theta_{t}}{2}\right)
\end{aligned}
$$

Similarly, symmetric imbalance on the receive-side implies $a_{r}^{I}=1+\tilde{\alpha}_{r}, a_{r}^{Q}=1-\tilde{\alpha}_{r}, \theta_{r}^{I}=-\theta_{r} / 2$, and $\theta_{r}^{Q}=\theta_{r} / 2$. Then, the expressions for $\mu_{r}$ and $\nu_{r}$ become

$$
\mu_{r}=\left(\cos \frac{\theta_{r}}{2}+j \tilde{\alpha}_{r} \sin \frac{\theta_{r}}{2}\right)
$$

$$
\nu_{r}=\left(\tilde{\alpha}_{r} \cos \frac{\theta_{r}}{2}-j \sin \frac{\theta_{r}}{2}\right)
$$

Therefore, in the symmetric imbalance case, the system performance depends on the amplitude differences $\tilde{\alpha}_{t}, \tilde{\alpha}_{r}$ and the phase differences $\theta_{t}, \theta_{r}$ between the I and Q branches, rather than on the actual imbalance parameters. The $\tilde{\alpha}$ 's are defined in log-scale as $\alpha_{t}=10 \log _{10}\left(1+\tilde{\alpha}_{t}\right) \mathrm{dB}$ and $\alpha_{r}=10 \log _{10}(1+$ $\left.\tilde{\alpha}_{r}\right) \mathrm{dB}$, so that $0 \mathrm{~dB}$ corresponds to no amplitude imbalance.

\section{B. Mobile OFDM With FD Joint Tx/Rx I/Q Imbalance}

In this section, we apply the I/Q imbalance model we developed in Section II-A to mobile OFDM systems. After sampling the receiver output, each of the analog quantities of the previous section, over one OFDM symbol duration, can be represented as a vector. The transmitted OFDM symbol, excluding cyclic prefix (CP), has $N$ samples of $\bar{s}(t)$, composing the vector $\bar{s}=[\bar{s}(0), \ldots, \bar{s}(N-1)]^{T}$. Likewise, the sampled version of $\bar{\lambda}_{t}(t)$ is given by $\bar{\lambda}_{t}=\left[\bar{\lambda}_{t}(0), \ldots, \bar{\lambda}_{t}\left(L_{\lambda_{t}}-1\right), \mathbf{0}_{1 \times\left(N-L_{\lambda_{t}}\right)}^{T}\right]^{T}$. Similarly, we define the vectors $\bar{\lambda}_{r}, \overline{\boldsymbol{\phi}}_{t}$, and $\overline{\boldsymbol{\phi}}_{r}$ associated with $\bar{\lambda}_{r}(t), \bar{\phi}_{t}(t)$, and $\bar{\phi}_{r}(t)$, respectively. Also, we sample the time-varying CIR at time $n$ and form $\bar{g}(n)=$ $\left[\bar{g}(n, 0), \ldots, \bar{g}(n, L-1), \mathbf{0}_{1 \times(N-L)}^{T}\right]^{T}$ for $n=0, \ldots, N-1$, where $L$ is the channel length. Assume that the combined response of the ideal transmit and receive filters $\left(\bar{h}_{r}(t) \otimes \bar{h}_{t}(t)\right)$ satisfies the Nyquist criterion. Also, assume that the $\mathrm{CP}$ length $N_{\mathrm{CP}}$ is higher than the memory lengths of $\bar{\psi}^{(1)}(t, \tau)$ and $\bar{\psi}^{(2)}(t, \tau)$ defined in (3). After removing CP, the linear convolutions in (3) become circular convolutions and the discrete-time received signal is given by (14). Collecting the $N$ samples of $\bar{z}(n)$ in the vector $\overline{\mathbf{z}}=[\bar{z}(0), \ldots, \bar{z}(N-1)]$, we can write $(15)$, where $\bar{\Lambda}_{t}$ is an $N \times N$ circulant matrix with its first column equal to $\overline{\boldsymbol{\lambda}}_{t}$. Similarly, the circulant matrices $\overline{\boldsymbol{\Lambda}}_{r}, \overline{\boldsymbol{\Phi}}_{t}$ and $\overline{\mathbf{\Phi}}_{r}$ are related to $\overline{\boldsymbol{\lambda}}_{r}, \overline{\boldsymbol{\phi}}_{t}$, and $\overline{\boldsymbol{\phi}}_{r}$, respectively. Under high mobility where the CIR varies within each OFDM symbol, $\overline{\mathbf{G}}$ is not a

$$
\begin{aligned}
& \bar{z}(n)=\left[\bar{\lambda}_{r}(n) \circledast_{N} \bar{g}(n, m) \circledast_{N} \bar{\lambda}_{t}(n)+\bar{\phi}_{r}(n) \circledast_{N} \bar{g}^{*}(n, m) \circledast_{N} \bar{\phi}_{t}^{*}(n)\right] \circledast_{N} \bar{s}(n) \\
& +\left[\bar{\lambda}_{r}(n) \circledast_{N} \bar{g}(n, m) \circledast_{N} \bar{\phi}_{t}(n)+\bar{\phi}_{r}(n) \circledast_{N} \bar{g}^{*}(n, m) \circledast_{N} \bar{\lambda}_{t}^{*}(n)\right] \circledast_{N} \bar{s}^{*}(n) \\
& +\bar{\lambda}_{r}(n) \otimes \bar{w}(n)+\bar{\phi}_{r}(n) \otimes \bar{w}^{*}(n) \text {. } \\
& \overline{\mathbf{z}}=\left(\overline{\mathbf{\Lambda}}_{r} \overline{\mathbf{G}} \overline{\mathbf{\Lambda}}_{t}+\overline{\mathbf{\Phi}}_{r} \overline{\mathbf{G}}^{*} \overline{\mathbf{\Phi}}_{t}^{*}\right) \overline{\mathbf{s}}+\left(\overline{\mathbf{\Lambda}}_{r} \overline{\mathbf{G}} \overline{\mathbf{\Phi}}_{t}+\overline{\mathbf{\Phi}}_{r} \overline{\mathbf{G}}^{*} \overline{\boldsymbol{\Lambda}}_{t}^{*}\right) \overline{\mathbf{s}}^{*} \\
& +\underbrace{\overline{\mathbf{\Lambda}}_{r}^{\prime} \overline{\mathbf{w}}+\overline{\boldsymbol{\Phi}}_{r}^{\prime} \overline{\mathbf{w}}^{*}}_{\overline{\mathbf{v}}} \rightarrow \text { Time Relation } \\
& \mathbf{z}=\underbrace{\left(\boldsymbol{\Lambda}_{r} \mathbf{G} \boldsymbol{\Lambda}_{t}+\boldsymbol{\Phi}_{r} \mathbf{G}^{\#} \boldsymbol{\Phi}_{t}^{\#}\right)}_{\boldsymbol{\Psi}^{(1)}} \mathbf{s} \\
& +\underbrace{\left(\boldsymbol{\Lambda}_{r} \mathbf{G} \boldsymbol{\Phi}_{t}+\boldsymbol{\Phi}_{r} \mathbf{G}^{\#} \boldsymbol{\Lambda}_{t}^{\#}\right)}_{\Psi^{(2)}} \mathbf{s}^{\#}+\mathbf{v} \rightarrow \text { Frequency Relation } \\
& \overline{\mathbf{G}}=\left[\begin{array}{ccccc}
\bar{g}(0,0) & \bar{g}(0, N-1) & \bar{g}(0, N-2) & \ldots & \bar{g}(0,1) \\
\bar{g}(1,1) & \bar{g}(1,0) & \bar{g}(1, N-1) & \ldots & \bar{g}(1,2) \\
\bar{g}(2,2) & \bar{g}(2,1) & \bar{g}(2,0) & \ldots & \bar{g}(2,3) \\
\vdots & \ddots & \ddots & \ddots & \vdots \\
\bar{g}(N-1, N-1) & \ldots & \ldots & \bar{g}(N-1,1) & \bar{g}(N-1,0)
\end{array}\right]
\end{aligned}
$$


circulant matrix but is given by (17), as shown at the bottom of the previous page. $\overline{\mathbf{w}}$ is the noise vector of length $\left(N+N_{\mathrm{CP}}\right)$. $\overline{\boldsymbol{\Lambda}}_{r}^{\prime}$ and $\overline{\boldsymbol{\Phi}}_{r}^{\prime}$ are matrices of size $N \times\left(N+N_{\mathrm{CP}}\right)$ whose $n$th rows are given by $\left[0_{1 \times n}, \lambda_{r}(0), \lambda_{r}(1), \ldots, \lambda_{r}\left(L_{\lambda_{r}}-1\right), \mathbf{0}\right]^{T}$, $\left[\mathbf{0}_{1 \times n}, \phi_{r}(0), \phi_{r}(1), \ldots, \phi_{r}\left(L_{\phi_{r}}-1\right), \mathbf{0}\right]^{T}$, respectively, and they represent linear convolutions for the noise components which have no cyclic prefix.

Applying the DFT to (15), we obtain the frequency-domain relation (16) where $\boldsymbol{\Lambda}_{t}=\mathbf{F} \overline{\boldsymbol{\Lambda}}_{t} \mathbf{F}^{H}, \boldsymbol{\Lambda}_{r}=\mathbf{F} \overline{\mathbf{\Lambda}}_{r} \mathbf{F}^{H}, \boldsymbol{\Phi}_{t}=$ $\mathbf{F} \overline{\boldsymbol{\Phi}}_{t} \mathbf{F}^{H}, \boldsymbol{\Phi}_{r}=\mathbf{F} \overline{\boldsymbol{\Phi}}_{r} \mathbf{F}^{H}, \mathbf{G}=\mathbf{F} \overline{\mathbf{G}} \mathbf{F}^{H}, \mathbf{z}=\mathbf{F} \overline{\mathbf{z}}, \mathbf{s}=\mathbf{F} \overline{\mathbf{s}}$, and $\mathbf{v}=\mathbf{F} \overline{\mathbf{v}}$. The $k$ th element of vector $\mathbf{b}^{\#}$ is the conjugate of the $(N-k)$ element of vector $\mathbf{b}$ and the $(n, k)$ element of matrix $\mathbf{B}^{\#}$ is the conjugate of the $(N-n, N-k)$ element of matrix $\mathbf{B}$, as shown at the bottom of the page.
The following remarks are in order.

- Since the matrices $\overline{\boldsymbol{\Lambda}}_{r}, \overline{\boldsymbol{\Lambda}}_{t}, \overline{\boldsymbol{\Phi}}_{r}$ and $\overline{\boldsymbol{\Phi}}_{t}$ are circulant, $\boldsymbol{\Lambda}_{r}$, matrices $\Lambda_{t}, \boldsymbol{\Phi}_{r}$ and $\boldsymbol{\Phi}_{t}$ are diagonal and so are $\boldsymbol{\Phi}_{t}^{\#}$ and $\boldsymbol{\Lambda}_{t}^{\#}$. Moreover, $\boldsymbol{\lambda}_{t}=\left[\lambda_{t}(0), \lambda_{t}(1), \ldots, \lambda_{t}(N-1)\right]^{T}=$ $\operatorname{Diag}\left(\boldsymbol{\Lambda}_{t}\right)$ represents the $N$-point DFT of $\bar{\lambda}_{t}$. The quantities $\boldsymbol{\lambda}_{r}, \boldsymbol{\phi}_{t}, \boldsymbol{\phi}_{r}, \boldsymbol{\lambda}_{t}^{\#}$ and $\boldsymbol{\phi}_{t}^{\#}$ are defined in a similar manner.

- Under high mobility, the CIR is no longer a time-invariant finite-impulse response (FIR) filter and $\overline{\mathbf{G}}$ is no longer circulant. In the frequency domain, the channel matrix $\mathbf{G}$ is no longer diagonal resulting in ICI. However, $\mathbf{G}$ can be well-approximated by a banded matrix as discussed in Section IV-B (see, e.g., [9] and references therein) since most of the ICI energy is due to neighboring sub-carriers.

$$
\begin{aligned}
& z(k)=\sum_{l=0}^{\frac{N}{2}-1}\left\{\left(\lambda_{r}(k) \lambda_{t}(l) g(k, l)+\phi_{r}(k) \phi_{t}^{*}\left(l^{\prime}\right) g^{*}\left(k^{\prime}, l^{\prime}\right)\right) s(l)\right. \\
& \left.+\left(\lambda_{r}(k) \phi_{t}\left(l^{\prime}\right) g\left(k, l^{\prime}\right)+\phi_{r}(k) \lambda_{t}^{*}(l) g^{*}\left(k^{\prime}, l\right)\right) s^{*}(l)\right\} \\
& +\sum_{m=0}^{\frac{N}{2}-1}\left\{\left(\lambda_{r}(k) \phi_{t}(m) g(k, m)+\phi_{r}(k) \lambda_{t}^{*}\left(m^{\prime}\right) g^{*}\left(k^{\prime}, m^{\prime}\right)\right) s^{*}\left(m^{\prime}\right)\right. \\
& \left.+\left(\lambda_{r}(k) \lambda_{t}\left(m^{\prime}\right) g\left(k, m^{\prime}\right)+\phi_{r}(k) \phi_{t}^{*}(m) g^{*}\left(k^{\prime}, m\right)\right) s\left(m^{\prime}\right)\right\}+v(k) \\
& z^{*}\left(k^{\prime}\right)=\sum_{l=0}^{\frac{N}{2}-1}\left\{\left(\lambda_{r}^{*}\left(k^{\prime}\right) \lambda_{t}^{*}(l) g^{*}\left(k^{\prime}, l\right)+\phi_{r}^{*}\left(k^{\prime}\right) \phi_{t}\left(l^{\prime}\right) g\left(k, l^{\prime}\right)\right) s^{*}(l)\right. \\
& \left.+\left(\lambda_{r}^{*}\left(k^{\prime}\right) \phi_{t}^{*}\left(l^{\prime}\right) g^{*}\left(k^{\prime}, l^{\prime}\right)+\phi_{r}^{*}\left(k^{\prime}\right) \lambda_{t}(l) g(k, l)\right) s(l)\right\} \\
& +\sum_{m=0}^{\frac{N}{2}-1}\left\{\left(\lambda_{r}^{*}\left(k^{\prime}\right) \phi_{t}^{*}(m) g^{*}\left(k^{\prime}, m\right)+\phi_{r}^{*}\left(k^{\prime}\right) \lambda_{t}\left(m^{\prime}\right) g^{*}\left(k, m^{\prime}\right)\right) s\left(m^{\prime}\right)\right. \\
& \left.+\left(\lambda_{r}^{*}\left(k^{\prime}\right) \lambda_{t}^{*}\left(m^{\prime}\right) g^{*}\left(k^{\prime}, m^{\prime}\right)+\phi_{r}^{*}\left(k^{\prime}\right) \phi_{t}(m) g(k, m)\right) s^{*}\left(m^{\prime}\right)\right\}+v^{*}\left(k^{\prime}\right) \\
& \boldsymbol{\gamma}_{k, l}^{(1)}=\left[\begin{array}{cc}
\lambda_{r}(k) \lambda_{t}(l) g(k, l)+\phi_{r}(k) \phi_{t}^{*}\left(l^{\prime}\right) g^{*}\left(k^{\prime}, l^{\prime}\right), & \lambda_{r}(k) \phi_{t}(l) g(k, l)+\phi_{r}(k) \lambda_{t}^{*}\left(l^{\prime}\right) g^{*}\left(k^{\prime}, l^{\prime}\right) \\
\lambda_{r}^{*}\left(k^{\prime}\right) \phi_{t}^{*}\left(l^{\prime}\right) g^{*}\left(k^{\prime}, l^{\prime}\right)+\phi_{r}^{*}\left(k^{\prime}\right) \lambda_{t}(l) g(k, l), & \lambda_{r}^{*}\left(k^{\prime}\right) \lambda_{t}^{*}\left(l^{\prime}\right) g^{*}\left(k^{\prime}, l^{\prime}\right)+\phi_{r}^{*}\left(k^{\prime}\right) \phi_{t}(l) g(k, l)
\end{array}\right] \\
& \underbrace{\left[\begin{array}{c}
\mathbf{z}_{0} \\
\vdots \\
\mathbf{z}_{\frac{N}{2}-1}
\end{array}\right]}_{\dot{z}}=\underbrace{\left[\begin{array}{ccc}
\boldsymbol{\gamma}_{0,0}^{(1)} & \cdots & \boldsymbol{\gamma}_{0, \frac{N}{2}-1}^{(1)} \\
\vdots & \ddots & \vdots \\
\boldsymbol{\gamma}_{\frac{N}{2}-1,0}^{(1)} & \cdots & \boldsymbol{\gamma}_{\frac{N}{2}-1, \frac{N}{2}-1}^{(1)}
\end{array}\right]}_{\boldsymbol{\Gamma}^{(1)}} \underbrace{\left[\begin{array}{c}
\mathbf{s}_{0} \\
\vdots \\
\mathbf{s}_{\frac{N}{2}-1}
\end{array}\right]}_{\dot{\boldsymbol{s}}} \\
& +\underbrace{\left[\begin{array}{ccc}
\boldsymbol{\gamma}_{0,0}^{(2)} & \cdots & \boldsymbol{\gamma}_{0, \frac{N}{2}-1}^{(2)} \\
\vdots & \ddots & \vdots \\
\boldsymbol{\gamma}_{\frac{N}{2}-1,0}^{(2)} & \cdots & \boldsymbol{\gamma}_{\frac{N}{2}-1, \frac{N}{2}-1}^{(2)}
\end{array}\right]}_{\boldsymbol{\Gamma}^{(2)}} \underbrace{\left[\begin{array}{c}
\mathbf{s}_{0}^{*} \\
\vdots \\
\mathbf{s}_{\frac{N}{2}-1}^{*}
\end{array}\right]}_{\boldsymbol{s}^{*}}+\underbrace{\left[\begin{array}{c}
\mathbf{v}_{0} \\
\vdots \\
\mathbf{v}_{\frac{N}{2}-1}
\end{array}\right]}_{\boldsymbol{v}} \\
& S(k)=\left(\lambda_{r}(k) \lambda_{t}(k) g(k, k)+\phi_{r}(k) \phi_{t}^{*}\left(k^{\prime}\right) g^{*}\left(k^{\prime}, k^{\prime}\right)\right) s(k) \\
& +\left(\lambda_{r}(k) \phi_{t}\left(k^{\prime}\right) g\left(k, k^{\prime}\right)+\phi_{r}(k) \lambda_{t}^{*}(k) g^{*}\left(k^{\prime}, k\right)\right) s^{*}(k) \\
& I(k)=\sum_{l=0, l \neq k}^{N-1}\left(\lambda_{r}(k) \lambda_{t}(l) g(k, l)+\phi_{r}(k) \phi_{t}^{*}\left(l^{\prime}\right) g^{*}\left(k^{\prime}, l^{\prime}\right)\right) s(l) \\
& +\left(\lambda_{r}(k) \phi_{t}(l) g(k, l)+\phi_{r}(k) \lambda_{t}^{*}\left(l^{\prime}\right) g^{*}\left(k^{\prime}, l^{\prime}\right)\right) s^{*}\left(l^{\prime}\right)
\end{aligned}
$$$$
\boldsymbol{\gamma}_{k, l}^{(2)}=\left[\begin{array}{cc}
\lambda_{r}(k) \phi_{t}\left(l^{\prime}\right) g\left(k, l^{\prime}\right)+\phi_{r}(k) \lambda_{t}^{*}(l) g^{*}\left(k^{\prime}, l\right), & \lambda_{r}(k) \lambda_{t}\left(l^{\prime}\right) g\left(k, l^{\prime}\right)+\phi_{r}(k) \phi_{t}^{*}(l) g^{*}\left(k^{\prime}, l\right) \\
\lambda_{r}^{*}(k) \lambda_{t}^{*}(l) g^{*}\left(k^{\prime}, l\right)+\phi_{r}^{*}\left(k^{\prime}\right) \phi_{t}\left(l^{\prime}\right) g\left(k, l^{\prime}\right), & \lambda_{r}^{*}\left(k^{\prime}\right) \phi_{t}^{*}(l) g^{*}\left(k^{\prime}, l\right)+\phi_{r}^{*}\left(k^{\prime}\right) \lambda_{t}\left(l^{\prime}\right) g\left(k, l^{\prime}\right)
\end{array}\right]
$$ 
- $\bar{g}(n, n-m)$ is the $(n, m)$ element of $\overline{\mathbf{G}}$. The $(k, l)$ element of $\mathbf{G}$ is $g(k, l)$, and it represents the interference due to the $l$ sub-carrier at the $k$ sub-carrier. From (17) and the fact that $\mathbf{G}=\mathbf{F} \overline{\mathbf{G}} \mathbf{F}^{H}$, it can be easily shown that the quantities $\bar{g}(n, n-m)$ and $g(k, l)$ are related as follows: ${ }^{1}$

$$
\begin{aligned}
g(k, l) & =\frac{1}{N} \sum_{n=0}^{N-1} e^{-j \frac{2 \pi}{N} k n} \sum_{m=0}^{N-1} \bar{g}(n, n-m) e^{j \frac{2 \pi}{N} m l} \\
& =\frac{1}{N} \sum_{n=0}^{N-1} e^{-j \frac{2 \pi}{N} k n} \sum_{m=0}^{N-1} \bar{g}(n, m) e^{j \frac{2 \pi}{N}(n-m) l}
\end{aligned}
$$

- The noise vector $\overline{\mathbf{v}}=\overline{\boldsymbol{\Lambda}}_{r}^{\prime} \overline{\mathbf{w}}+\overline{\mathbf{\Phi}}_{r}^{\prime} \overline{\mathbf{w}}^{*}$, after being subject to the receiver FI I/Q imbalance, is no longer white and its covariance matrix is given by (59).

\section{Equivalent Channel Matrix}

This section develops an alternative and more compact matrix representation for (16) which sheds light on the distinctive features of ICI due to I/Q imbalance and mobility. First, the $k$ and $k^{\prime}=N-k$ elements of $\mathbf{z}$ in (16) are written down in a special form as shown in (18) and (19), where $l^{\prime}=N-l, m^{\prime}=N-m$ and $k=0, \ldots,(N / 2)-1$. In these summations, each term contains both $s(l)$ and $s^{*}(l)$. This is because, due to mobility, each signal term $s(l)$ on sub-carrier $l$ leaks to other sub-carriers including $l^{\prime}$. I/Q imbalance, on the other hand, reflects back a conjugate image version of the leaked component at $l^{\prime}$, namely $s^{*}(l)$. Such a self-interference scenario occurs only in the presence of both I/Q imbalance and mobility. For each sub-carrier pair $\left(k, k^{\prime}\right),(18)$ and (19) could be combined and written in matrix form as

$$
\mathbf{z}_{k}=\sum_{l=0}^{\frac{N}{2}-1}\left(\gamma_{k, l}^{(1)} \mathbf{s}_{l}+\boldsymbol{\gamma}_{k, l}^{(2)} \mathbf{s}_{l}^{*}\right)+\mathbf{v}_{k}
$$

where $\mathbf{z}_{k}=\left[z(k), z^{*}\left(k^{\prime}\right)\right]^{T}, \mathbf{s}_{l}=\left[s(l), s^{*}\left(l^{\prime}\right)\right]^{T}, \mathbf{v}_{k}=$ $\left[v(k), v^{*}\left(k^{\prime}\right)\right]^{T}$, and $\boldsymbol{\gamma}_{k, l}$ 's are given in (20). The self-interference effect is caught in the matrix $\gamma_{k, l}^{(2)}$. The terms in (25) could be collected for all $k$ as in (21). In the special case of no mobility, only $g(k, l)$ 's for $k=l$ are nonzero and hence only those $\gamma_{k, l}^{(1)}$, s with $k=l$ are nonzero, i.e., $\boldsymbol{\Gamma}^{(1)}$ becomes a block-diagonal matrix. Furthermore, $\boldsymbol{\Gamma}^{(2)}$ vanishes and so (21) decouples into $(N / 2)$ subsystems of size $2 \times 2$. If we further specialize to the FI I/Q imbalance case, i.e., $\phi_{r}(k)=\mu_{r}, \phi_{t}(k)=\mu_{t}, \lambda_{r}(k)=\nu_{r}, \lambda_{t}(k)=\nu_{t} \forall k$, then (21) reduces to the model in [4].

\section{SINR ANALYSIS}

In this section, we present new insights on the combined ICI effects of mobility and I/Q imbalance by analyzing the resulting degradation in the SINR. We make the following assumptions.

1) Channel $\bar{g}(n, m)$ is uncorrelated for different taps $m$. For each $m,\{\bar{g}(n, m): n=0, \ldots, N-1\}$ is a circularlysymmetric complex Gaussian vector.

2) $s(k)$ is a circularly symmetric complex random vector with average energy $E_{s}=E\left\{|s(k)|^{2}\right\}$.

${ }^{1}$ The form in (24) is obtained by simple rearrangement and is useful in SINR analysis.
3) $\sum_{m=0}^{L-1} \sigma_{\bar{g}_{m}}^{2}=1$ where $\sigma_{\bar{g}_{m}}^{2}$ is the power of tap- $m$, i.e., the channel results in no net gain.

Along with these assumptions, we split the terms in (18) into two parts, one being the signal term with $l=k$ and the other involving the ICI terms with $l \neq k$, and rewrite as follows:

$$
z(k)=S(k)+I(k)+v(k)
$$

where $S(k)$ and $I(k)$ are defined in (22) and (23), respectively. In the Appendix, we derive the expressions for $E\left\{|S(k)|^{2}\right\}$ and $E\left\{|I(k)|^{2}\right\}$ for the generalized case and also the variance of the colored noise. For the special case of FI I/Q imbalance, they can be simplified as shown in (27)-(29), where $R\left(n_{1}, n_{2}\right)=$ $E\left\{\bar{g}\left(n_{1}, m\right) \bar{g}^{*}\left(n_{2}, m\right)\right\}$ is the normalized time-correlation of CIR tap $m$. Finally, the SINR and signal-to-interference ratio (SIR) are defined as follows:

$$
\begin{aligned}
\mathrm{SINR}= & \frac{E\left\{|S(k)|^{2}\right\}}{E\left\{|I(k)|^{2}\right\}+E\left\{|v(k)|^{2}\right\}} \\
\mathrm{SIR}= & \frac{E\left\{|S(k)|^{2}\right\}}{E\left\{|I(k)|^{2}\right\}} \\
E\left\{|S(k)|^{2}\right\} & \frac{1}{N^{2}} E_{s}\left[\left(\left|\mu_{r}\right|^{2}\left|\mu_{t}\right|^{2}+\left|\nu_{r}\right|^{2}\left|\nu_{t}\right|^{2}\right) \sum_{n_{1}, n_{2}} R\left(n_{1}, n_{2}\right)\right. \\
& +\left|\mu_{r}\right|^{2}\left|\nu_{t}\right|^{2} \sum_{n_{1}, n_{2}} R\left(n_{1}, n_{2}\right) e^{-j \frac{4 \pi}{N}\left(n_{1}-n_{2}\right) k} \\
& \left.+\left|\nu_{r}\right|^{2}\left|\mu_{t}\right|^{2} \sum_{n_{1}, n_{2}} R\left(n_{1}, n_{2}\right) e^{+j \frac{4 \pi}{N}\left(n_{1}-n_{2}\right) k}\right] \\
E\left\{|I(k)|^{2}\right\} & \\
= & \frac{E_{s}}{N^{2}}\left[\left(\left|\mu_{r}\right|^{2}\left|\mu_{t}\right|^{2}+\left|\nu_{r}\right|^{2}\left|\nu_{t}\right|^{2}\right)\right. \\
& \times\left(N^{2}-\sum_{n_{1}, n_{2}} R\left(n_{1}, n_{2}\right)\right) \\
& +\left|\mu_{r}\right|^{2}\left|\nu_{t}\right|^{2}\left(N^{2}-\sum_{n_{1}, n_{2}} R\left(n_{1}, n_{2}\right) e^{-j \frac{4 \pi}{N}\left(n_{1}-n_{2}\right) k}\right) \\
& +\left|\nu_{r}\right|^{2}\left|\mu_{t}\right|^{2}\left(N^{2}-\sum_{n_{1}, n_{2}} R\left(n_{1}, n_{2}\right) e^{+j \frac{4 \pi}{N}\left(n_{1}-n_{2}\right) k}\right) \\
E\left\{|v(k)|^{2}\right\} & \left(\left|\mu_{r}\right|^{2}+\left|\nu_{r}\right|^{2}\right) \sigma^{2} .
\end{aligned}
$$

Case Study: Assume that the time-varying CIR obeys the Jake's model [10], i.e., $R\left(n_{1}, n_{2}\right)=J_{0}\left(2 \pi f_{D}\left(n_{2}-n_{1}\right) T\right)$, where $J_{0}(\cdot)$ is the zero-order Bessel function and $f_{D}$ is the maximum Doppler frequency. Then, using the symmetries of the Bessel function, we obtain (30) and (31). Substituting these into (27) and (28), we obtain the corresponding SINR and SIR expressions. In (30) and (31), we found that the value of $R_{2} \ll$ $R_{1}$. Therefore, we ignore $R_{2}$ in the following results and we consider the case of FI I/Q imbalance. Fig. 3 compares the SINR under mild and severe $\mathrm{I} / \mathrm{Q}$ imbalance and mobility scenarios. It can be seen that when the I/Q imbalance parameters change from $\left(\alpha_{t}=\alpha_{r}, \theta_{t}=\theta_{r}\right)=\left(0.1 \mathrm{~dB}, 1^{\circ}\right)$, to $\left(0.5 \mathrm{~dB}, 5^{\circ}\right)$, 


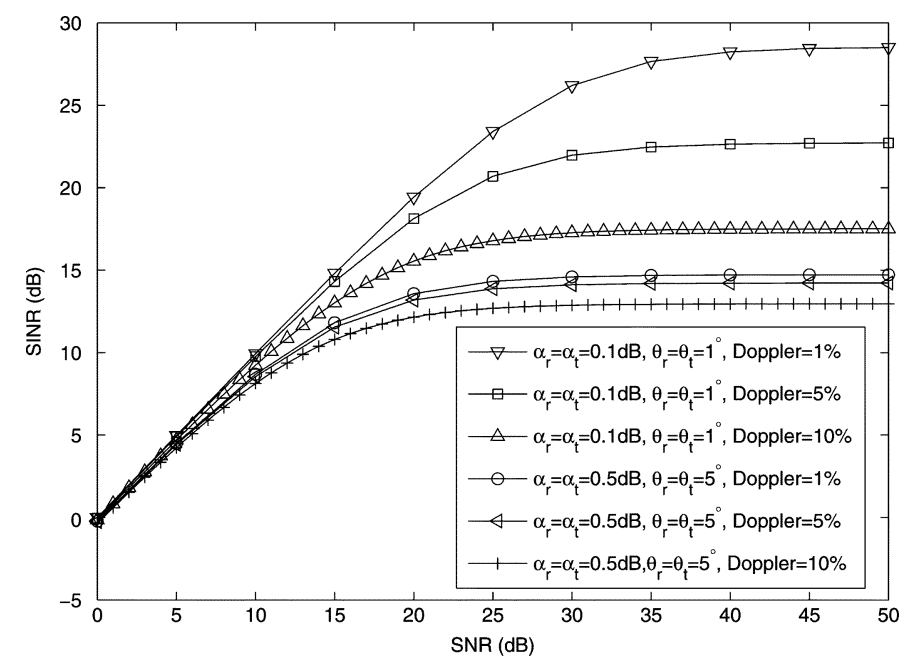

Fig. 3. SINR comparison with different I/Q imbalance and Doppler parameters.

the performance degrades dramatically. An interesting observation from Fig. 3 is that at low I/Q imbalance $\left(0.1 \mathrm{~dB}, 1^{\circ}\right)$, as Doppler increases (from $1 \%$ to $5 \%$ to $10 \%$ ), the SINR degrades significantly (from $28.5 \mathrm{~dB}$ to $22.7 \mathrm{~dB}$ to $14.2 \mathrm{~dB}$ ) while at high I/Q imbalance $\left(0.5 \mathrm{~dB}, 5^{\circ}\right)$, the SINR performance only degrades slightly (from $14.7 \mathrm{~dB}$ to $14.2 \mathrm{~dB}$ to $12.9 \mathrm{~dB}$ ). Thus, when I/Q imbalance is mild, Doppler spread has larger impact on the SINR performance and vice versa.

$$
\begin{aligned}
& \sum_{n_{1}=0}^{N-1} \sum_{n_{2}=0}^{N-1} R\left(n_{1}, n_{2}\right) \\
& \quad=\sum_{n_{1}=0}^{N-1} \sum_{n_{2}=0}^{N-1} J_{0}\left(2 \pi f_{D}\left(n_{2}-n_{1}\right) T\right) \\
& \quad=N+2 \sum_{n=1}^{N-1}(N-n) J_{0}\left(2 \pi f_{D} n T\right) \triangleq R_{1} \\
& \sum_{n_{1}=0}^{N-1} \sum_{n_{2}=0}^{N-1} R\left(n_{1}, n_{2}\right) e^{-j \frac{2 \pi}{N}(2 k)\left(n_{1}-n_{2}\right)} \\
& \quad=N+2 \sum_{n=1}^{N-1}(N-n) J_{0}\left(2 \pi f_{D} n T\right) \cos \left(\frac{4 \pi k n}{N}\right) \triangleq R_{2}
\end{aligned}
$$

SIR

$$
=\frac{\left(\left|\eta_{r}\right|^{2}\left|\eta_{t}\right|^{2}+1\right) R_{1}+\left(\left|\eta_{r}\right|^{2}+\left|\eta_{t}\right|^{2}\right) R_{2}}{\left(\left|\eta_{r}\right|^{2}\left|\eta_{t}\right|^{2}+1\right)\left(N^{2}-R_{1}\right)+\left(\left|\eta_{r}\right|^{2}+\left|\eta_{t}\right|^{2}\right)\left(N^{2}-R_{2}\right)} .
$$

To gain more insight into the impact of different I/Q imbalance parameters, we define $\eta_{r}=\left(\mu_{r}\right) /\left(\nu_{r}\right)$ and $\eta_{t}=\left(\mu_{t}\right) /\left(\nu_{t}\right)$ using which we can write the SIR as (32). Fig. 4 shows the variation of SIR against $\eta_{r}=\eta_{t}=\eta$ for different Doppler spreads. The vertical dashed lines highlight mild and severe I/Q imbalances. It can be clearly seen that SIR increases with $\eta$ until it reaches its Doppler-limited level. When $\eta_{r}=\eta_{t}=\infty$, there is no I/Q imbalance and $\mathrm{SIR}=\left(R_{1}\right) /\left(N^{2}-R_{1}\right)$, in agreement

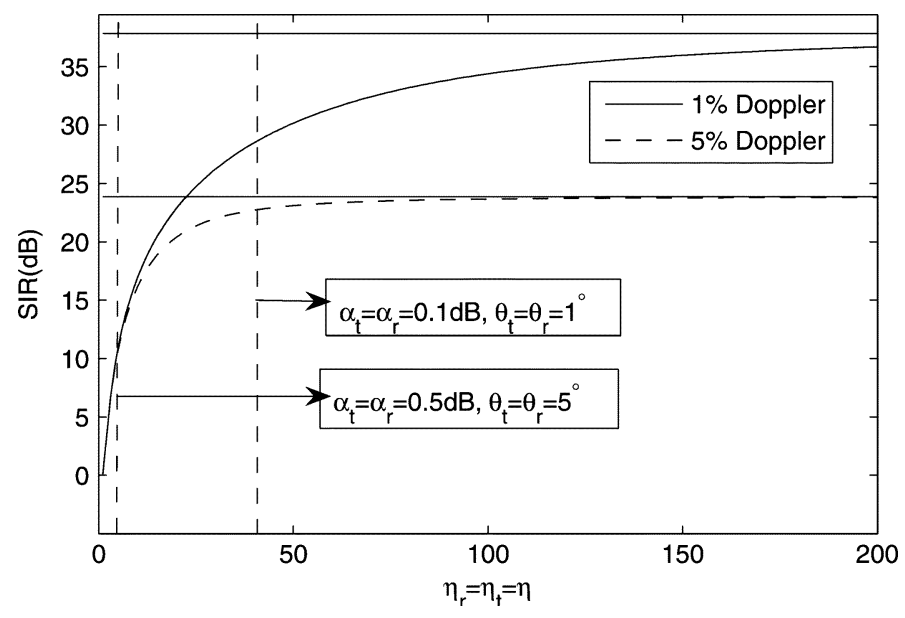

Fig. 4. SIR variation with different $\mathrm{I} / \mathrm{Q}$ imbalance parameters.

with the result in [10]. These results clearly illustrate the importance of compensating for both I/Q imbalance and mobility, which is the subject of the next section.

\section{Digital BASEBAND COMPENSATION}

In Section III, we have shown that both severe I/Q imbalance and high mobility degrade the SINR performance dramatically. In this section, we propose joint compensation schemes for the impairments. First, we investigate the block MMSE frequencydomain equalizer (MMSE-FEQ) which is high in complexity. Then, we revisit the structure of the channel matrix and exploit some of its properties to derive a reduced-complexity symbolwise FIR MMSE-FEQ.

\section{A. Block MMSE-FEQ}

The signal model in (21) has both $s(k)$ and $s^{*}(k)$ terms in addition to colored noise due to FD I/Q imbalance. We split the real and imaginary terms of the complex system in (21) as

$$
\underbrace{\left[\begin{array}{c}
\Re\left\{\mathbf{z}^{\prime}\right\} \\
\Im\{\mathbf{z}\}
\end{array}\right]}_{\mathbf{z}_{\mathrm{IQ}}}=\boldsymbol{\Gamma}_{\mathrm{IQ}} \underbrace{\left[\begin{array}{c}
\Re\{\dot{\mathbf{s}}\} \\
\Im\left\{\mathbf{s}^{\prime}\right\}
\end{array}\right]}_{\mathbf{s}_{\mathrm{IQ}}}+\underbrace{\left[\begin{array}{c}
\Re\{\dot{\mathbf{v}}\} \\
\Im\{\mathbf{v}\}
\end{array}\right]}_{\mathbf{v}_{\mathrm{IQ}}}
$$

where

$$
\boldsymbol{\Gamma}_{\mathrm{IQ}}=\left[\begin{array}{cc}
\Re\left\{\boldsymbol{\Gamma}^{(1)}\right\} & -\Im\left\{\boldsymbol{\Gamma}^{(1)}\right\} \\
\Im\left\{\boldsymbol{\Gamma}^{(1)}\right\} & \Re\left\{\boldsymbol{\Gamma}^{(1)}\right\}
\end{array}\right]+\left[\begin{array}{cc}
\Re\left\{\boldsymbol{\Gamma}^{(2)}\right\} & \Im\left\{\boldsymbol{\Gamma}^{(2)}\right\} \\
\Im\left\{\boldsymbol{\Gamma}^{(2)}\right\} & -\Re\left\{\boldsymbol{\Gamma}^{(2)}\right\}
\end{array}\right] .
$$

For simplicity, assuming the noise to be white, the Block MMSE-FEQ is given by

$$
\hat{\mathbf{s}}_{\mathrm{IQ}}=\boldsymbol{\Gamma}_{\mathrm{IQ}}^{H}\left(\boldsymbol{\Gamma}_{\mathrm{IQ}} \boldsymbol{\Gamma}_{\mathrm{IQ}}^{H}+\frac{1}{\mathrm{SNR}_{2 N}}\right)^{-1} \mathbf{z}_{\mathrm{IQ}} .
$$

However, the calculation of (34) involves the inversion of a real matrix of size $2 N$ and is of high complexity. In the following, we will present a reduced-complexity FIR-MMSE FEQ which exploits the banded structure of the $\mathbf{G}$ matrix [see (17)], as shown in (35) at the top of the next page.

\section{B. Reduced-Complexity FIR MMSE-FEQ}

As previously discussed in Section II, $\boldsymbol{\Lambda}_{r}, \boldsymbol{\Lambda}_{t}, \boldsymbol{\Phi}_{r}, \boldsymbol{\Phi}_{t}, \boldsymbol{\Lambda}_{t}^{\#}$, and $\boldsymbol{\Phi}_{t}^{\#}$ in (16) are diagonal matrices. $\mathbf{G}$ is not diagonal in general and this results in a computationally-complex equalizer as 


$$
\tilde{\mathbf{G}}(k)=\left[\begin{array}{ccccccc}
\boldsymbol{\gamma}_{k-D, k-2 D}^{(1)} & \ldots & \boldsymbol{\gamma}_{k-D, k-D}^{(1)} & \cdots & \boldsymbol{\gamma}_{k-D, k}^{(1)} & & \\
& \boldsymbol{\gamma}_{k, k-D}^{(1)} & \ldots & \boldsymbol{\gamma}_{k, k}^{(1)} & \ldots & \boldsymbol{\gamma}_{k, k+D}^{(1)} & \\
& & \boldsymbol{\gamma}_{k+D, k}^{(1)} & \cdots & \boldsymbol{\gamma}_{k+D, k+D}^{(1)} & \cdots & \boldsymbol{\gamma}_{k+D, k+2 D}^{(1)}
\end{array}\right]
$$

TABLE I

PERCENTAGE Distribution OF ENERGy IN $\boldsymbol{\Gamma}^{(1)}$ AND $\boldsymbol{\Gamma}^{(2)}$ (ASSUMED I/Q IMBALANCE PARAMETERS: $1 \mathrm{~dB}, 5^{\circ}$ )

\begin{tabular}{|c|c|c|c|c|c|c|}
\hline & \multicolumn{5}{|c|}{$\Gamma^{(1)}$} & $\Gamma^{(2)}$ \\
\hline Doppler & Dg=0 & Dg=1 & Dg=2 & Dg=3 & D $>3$ & All \\
\hline $5 \%$ & 99.25 & 0.45 & 0.11 & 0.05 & $<0.03$ & $<0.04$ \\
\hline $10 \%$ & 97.7 & 1.4 & 0.35 & 0.15 & $<0.1$ & $<0.08$ \\
\hline
\end{tabular}

in (34), but, as shown in [9] and references therein, $\mathbf{G}$ can be well approximated by a banded matrix as most of the ICI energy is from neighboring sub-carriers. The number of significant diagonals of $\mathbf{G}$ depends on the Doppler spread. Assume that we only consider $Q$ diagonals in the banded matrix $\mathbf{G}$ and set $Q=2 D+1$. With this banded $\mathbf{G}$, we follow the same steps described in Section II-C to arrive at the following $2 \times 2$ subsystems for $0 \leq k \leq(N / 2)$

$\mathbf{z}_{k}=\boldsymbol{\gamma}_{k, k}^{(1)} \mathbf{s}_{k}+\sum_{\substack{i=-D \\ i \neq 0}}^{D} \boldsymbol{\gamma}_{k, k-i}^{(1)} \mathbf{s}_{k-i}+\sum_{i=-D}^{D} \boldsymbol{\gamma}_{k, k-i}^{(2)} \mathbf{s}_{k-i}^{*}+\mathbf{v}_{\mathbf{k}}$

where the $\gamma_{k, l}$ 's were defined (20). Equation (36) can also be derived from (21) by setting all the sub-blocks $\gamma_{k, l}^{(1)}=\mathbf{0}_{2 \times 2}$ for $|k-l|>D$. The same condition also annuls most blocks of $\gamma_{k, l}^{(2)}$. Shown in Table I are the energies of the diagonals of $\boldsymbol{\Gamma}^{(1)}$ in a 512 sub-carrier 5-MHz OFDM system using the Stanford University Interim-3 (SUI-3) Channel model. It can be seen that most of the energy of the equivalent channel matrix is concentrated in its main diagonal $(\mathrm{Dg}=0)$. Less than $1 \%$ of the energy spills into the sub-diagonals $(\mathrm{Dg} \geq 1)$ at $5 \%$ Doppler and less than $2.5 \%$ at $10 \%$ Doppler. Also, the energy of the whole matrix $\boldsymbol{\Gamma}^{(2)}$ is negligible.

With the banded matrix approximation justified, as a performance complexity tradeoff, we consider an FIR MMSE-FEQ structure [11] with $2 Q$ taps per sub-carrier ${ }^{2}$, where $Q$ accounts for the adjacent sub-carriers and the factor 2 accounts for the image sub-carriers. To compute the optimum FEQ tap settings, based on (36), we define the quantities $\tilde{\mathbf{z}}_{k} \triangleq\left[\mathbf{z}_{k-D}^{T}, \ldots, \mathbf{z}_{k}^{T}, \ldots, \mathbf{z}_{k+D}^{T}\right]^{T}$, $\tilde{\mathbf{s}}_{k} \triangleq \quad\left[\mathbf{s}_{k-2 D}^{T}, \ldots, \mathbf{s}_{k}^{T}, \ldots, \mathbf{s}_{k-2 D}^{T}\right]^{T}, \quad \tilde{\mathbf{v}}_{k} \triangleq$ $\left[\mathbf{v}_{k-D}^{T}, \ldots, \mathbf{v}_{k}^{T}, \ldots, \mathbf{v}_{k+D}^{T}\right]^{T}$, and $\tilde{\mathbf{G}}(k)$ as in (35).

Then, we have $\tilde{\mathbf{z}}_{k}=\widetilde{\mathbf{G}}_{k} \tilde{\mathbf{s}}_{k}+\tilde{\mathbf{v}}_{k}$ and the linear MMSE estimator for $\mathbf{s}_{k}=\left[s(k), s^{*}(N-k)\right]^{T}$ is $\hat{\mathbf{s}}_{k}=\mathbf{w}_{k}^{H} \tilde{\mathbf{z}}_{k}$ where

$$
\mathbf{w}_{k}^{H}=\tilde{\mathcal{G}}_{k}^{H}\left(\tilde{\mathbf{G}}_{k} \tilde{\mathbf{G}}_{k}^{H}+\sigma^{2} \mathbf{I}_{2 Q}\right)^{-1}
$$

${ }^{2}$ This holds for $k=D$ to $(N / 2)-D-1$. For sub-carriers outside this range which fall closer to the edges, the number of taps decreases progressively.
TABLE II

COMPUTATIONAL COMPLEXITY (IN FLOPS) FOR DIFFERENT COMPENSATION SCENARIOS

\begin{tabular}{|c|c|c|c|}
\hline$N \backslash$ taps & $Q=3$ & $Q=5$ & $Q=N$ \\
\hline 512 & $2.38 \times 10^{6}$ & $1.10 \times 10^{7}$ & $5.73 \times 10^{9}$ \\
\hline 1024 & $4.77 \times 10^{6}$ & $2.19 \times 10^{7}$ & $4.58 \times 10^{10}$ \\
\hline
\end{tabular}

and $\tilde{\mathcal{G}}_{k}$ is the middle block column of $\tilde{\mathbf{G}}_{k}$. Note that we detect the $k$ th sub-carrier $s(k)$ and its image $s^{*}(N-k)$ jointly. Since $Q \ll N$, significant complexity reductions are achieved in comparison with the block FIR MMSE-FEQ in Section IV-A at negligible performance loss.

\section{Complexity Analysis}

First, we determine the number of computations required for the $N$-tap FEQ in (34). From [12], we have for a real square matrix of size- $n, 2 n^{3}$ flops for multiplication, For this, we use the following results from [12] for real square $m$.

- Multiplying two real matrices of sizes $m \times n$ and $n \times r$ requires $2 m n r$ flops.

- Gaussian elimination of a real square matrix of size $n$ requires $\left(2 n^{3} / 3\right)$ flops.

- Matrix inversion of a real square matrix involves two Gaussian elimination steps and hence requires $\left(4 n^{3} / 3\right)$ flops.

Here, a flop is defined as either an addition or multiplication operation. Therefore, an $N$-tap FEQ requires $8 N^{2}+\left(128 N^{3} / 3\right)$ flops. Also, we evaluate the complexity involved in computing the $(Q=2 D+1)$-tap FEQ in (37). This turns out to be $(16 N / 3)(1+2 D)(35+128 D(1+D))$ flops and is evaluated in Table II for different values of $N$ and $D$. Clearly, our proposed compensation schemes results in significant complexity reductions.

\section{Channel Estimation Schemes}

To implement the FIR MMSE-FEQ proposed in Section IV-B, effective channel estimation schemes are essential. In this section, we first propose a pilot pattern which can be used for channel estimation with both FI and FD I/Q imbalances. Next, for the FI I/Q imbalance case, we propose a low-overhead pilot pattern by computing I/Q imbalance parameter ratios $\eta_{r}$ and $\eta_{t}$.

\section{A. Scheme A: General Pilot Scheme for FI/FD I/Q Imbalance}

The proposed pilot pattern is shown in Fig. 5. In this scheme, denoted by Scheme A, two pairs of pilots are placed on the sub-carriers $(k, N-k)$ and $(k+1, N-k-1)$. In most practical OFDM systems, the coherence bandwidth is much larger than a pair of adjacent sub-carriers. Thus, it is reasonable to assume that sub-carriers $k$ and $(k+1)$ have the same frequency response and so do sub-carriers $(N-k-1)$ and $(N-k)$. To 


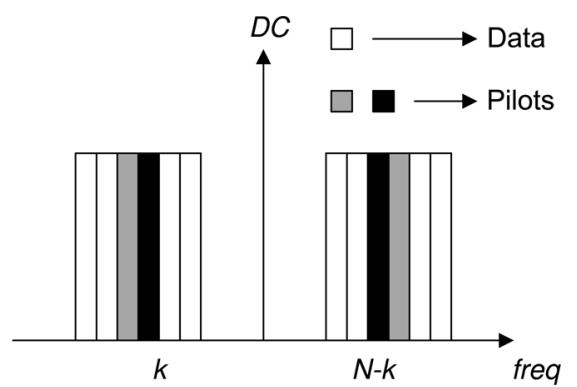

Fig. 5. Pilot pattern.

proceed with further development, we introduce the following useful notations:

$$
\gamma_{k, k}^{(1)}=\left[\begin{array}{ll}
h_{k}(1) & h_{k}(2) \\
h_{k}(3) & h_{k}(4)
\end{array}\right]
$$

and vector $\mathbf{h}_{k}=\left[h_{k}(1) h_{k}(2) h_{k}(3) h_{k}(4)\right]^{T}$. With respect to (16), it should be noted that $h_{k}(1)$ and $h_{k}(2)$ are the $(k, k)$ elements of $\boldsymbol{\Psi}^{(1)}$ and $\boldsymbol{\Psi}^{(2)}$, respectively, and $h_{k}^{*}(4)$ and $h_{k}^{*}(3)$ are the $(N-k, N-k)$ elements. Using the assumption, new notations, and ignoring $\boldsymbol{\gamma}_{k, l}^{(2)}$, s, (36) for $\mathbf{z}_{k}$ and $\mathbf{z}_{k+1}$ can be combined and rewritten as

$$
\begin{aligned}
\underbrace{\left[\begin{array}{c}
\mathbf{z}_{k} \\
\mathbf{z}_{k+1}
\end{array}\right]}_{\mathbf{z}_{k}^{\prime}}= & \underbrace{\left[\begin{array}{cccc}
s(k) & s^{*}\left(k^{\prime}\right) & 0 & 0 \\
0 & 0 & s(k) & s^{*}\left(k^{\prime}\right) \\
s(k+1) & s^{*}\left(k^{\prime}-1\right) & 0 & 0 \\
0 & 0 & s(k+1) & s^{*}\left(k^{\prime}-1\right)
\end{array}\right]}_{\mathbf{S}_{k}} \\
& \times \mathbf{h}_{k}+\mathrm{ICI}+\left[\begin{array}{c}
\mathbf{v}_{k} \\
\mathbf{v}_{k+1}
\end{array}\right] .
\end{aligned}
$$

The ICI and noise could be lumped together and assuming this lumped noise to be white, the Best Linear Unbiased Estimator (BLUE) [13] for $\mathbf{h}_{k}$ is given by

$$
\hat{\mathbf{h}}_{k}=\mathbf{S}_{k}^{-1} \mathbf{z}_{k}^{\prime} \text {. }
$$

Moreover, we chose $\mathbf{S}_{k}$ to be an orthogonal matrix to achieve minimum variance for the estimator. If we further restrict the pilots to be BPSK symbols, a choice for $\mathbf{S}_{k}$ would be $\left\{s(k), s\left(k^{\prime}\right), s(k+1), s\left(k^{\prime}-1\right)\right\}=\{1,1,1,-1\}$. In this process, we assume that the ICI caused by the adjacent data sub-carriers could be ignored since the energy of the pilots is typically boosted compared to the data as in the WiMAX standard [14]. Moreover, estimating all the $\mathbf{h}_{k}$ 's yields only the diagonal blocks $\boldsymbol{\gamma}_{k, k}^{(1)}$, s. To obtain the off-diagonal elements, we use the approach presented in [15].

\section{B. Scheme B: Pilot Scheme for FI I/Q Imbalance}

For FI I/Q imbalance we have $\phi_{r}(k)=\mu_{r}, \phi_{t}(k)=$ $\mu_{t}, \lambda_{r}(k)=\nu_{r}$ and $\lambda_{t}(k)=\nu_{t} \forall k$. If we define $\beta(k)=\mu_{r} \mu_{t} g(k, k)$ and $\beta(N-k)=\mu_{r} \mu_{t} g(N-k, N-k)$, we can express $\mathbf{h}_{k}$ 's elements in terms of the I/Q imbalance parameters ratios, $\eta_{t}=\left(\mu_{t}\right) /\left(\nu_{t}\right)$ and $\eta_{r}=\left(\mu_{r}\right) /\left(\nu_{r}\right)$, as follows:

$$
h_{k}(1)=\beta(k)+\frac{1}{\eta_{r}} \frac{1}{\eta_{t}^{*}} \beta^{*}(N-k)
$$

$$
\begin{aligned}
& h_{k}(2)=\frac{1}{\eta_{t}} \beta(k)+\frac{1}{\eta_{r}} \beta^{*}(N-k) \\
& h_{k}(3)=\frac{1}{\eta_{r}^{*}} \beta(k)+\frac{1}{\eta_{t}^{*}} \beta^{*}(N-k) \\
& h_{k}(4)=\frac{1}{\eta_{r}^{*}} \frac{1}{\eta_{t}} \beta(k)+\beta^{*}(N-k) .
\end{aligned}
$$

Now, if $\eta_{t}$ and $\eta_{r}$ are known, then there are only two unknowns $\beta(k)$ and $\beta(N-k)$ in system (40) and hence only a pair of pilot sub-carriers $(k, N-k)$ would be required to estimate them, as against Scheme A which requires two pairs to solve for four unknowns. This, we call Scheme B, but first, we have to estimate the ratios $\eta_{t}$ and $\eta_{r}$. For this, we use a mixed pilot structure where at least one OFDM symbol in a frame (such as the preamble) has the pilot structure of Scheme A. The rest of the OFDM symbols in the frame need to have only the pilots shown in gray in Fig. 5 and the pilots shown in black can be replaced with data reducing the overhead by a factor of two.

Now, we describe the procedure for estimating $\eta_{t}$ and $\eta_{r}$ using system (40) (that results from the preamble) which is nonlinear in $\left(1 / \eta_{t}\right),\left(1 / \eta_{r}\right), \beta(k)$ and $\beta(N-k)$, and has no straightforward solution. However, multiplying (40c) by $\left(1 / \eta_{t}\right)$ and subtracting (40d) from it, we obtain

$$
\beta^{*}(N-k)=\frac{\frac{1}{\eta_{t}} h_{k}(3)-h_{k}(4)}{\frac{1}{\left|\eta_{t}\right|^{2}}-1} .
$$

Similarly, multiplying (40b) with $\left(1 / \eta_{t}^{*}\right)$ and subtracting (40a) from it, we obtain

$$
\beta(k)=\frac{\frac{1}{\eta_{t}^{*}} h_{k}(2)-h_{k}(1)}{\frac{1}{\left|\eta_{t}\right|^{2}}-1} .
$$

Then, substituting (41) and (42) into (40b), we obtain

$$
\frac{1}{\eta_{r}}=\frac{\frac{1}{\eta_{t}} h_{k}(1)-h_{k}(2)}{\frac{1}{\eta_{t}} h_{k}(3)-h_{k}(4)} .
$$

Finally, substituting (41), (42) and (43) into (40c), we obtain the following quadratic equation:

$$
\begin{aligned}
& \left(\frac{1}{\eta_{t}^{*}}\right)^{2}(\underbrace{h_{k}^{*}(1) h_{k}(2)-h_{k}(4) h_{k}^{*}(3)}_{a}) \\
& +\frac{1}{\eta_{t}^{*}}(\underbrace{\left|h_{k}(3)\right|^{2}+\left|h_{k}(4)\right|^{2}-\left|h_{k}(1)\right|^{2}-\left|h_{k}(2)\right|^{2}}_{b}) \\
& +\underbrace{h_{k}(1) h_{k}^{*}(2)-h_{k}^{*}(4) h_{k}(3)}_{c}=0 .
\end{aligned}
$$

Note that $a=c^{*}$. If $a=0$, then $\left(1 / \eta_{t}\right)=0$. Otherwise, there are two roots for (44) and the absolute value of the product of the two roots is 1 since $a=c^{*}$. Since $\left|\eta_{t}\right|>1$ in practical systems, we choose the root of (44) which satisfies $\left|1 / \eta_{t}\right|<1$. Similarly the value of $\left(1 / \eta_{r}\right)$ is found. These are then used to estimate the channel frequency responses at the pilot positions in OFDM symbols other than the preamble.

\section{Simulation Results}

In this section, we present the simulation results for our proposed compensation and channel estimation schemes under different operating scenarios. 


\section{A. Simulation Setup}

The system parameters are similar to the 512 sub-carrier profile of the 802.16e mobile WiMAX standard [14], i.e., the bandwidth is $5 \mathrm{MHz}$, the sampling frequency is $5.6 \mathrm{MHz}$ and the operating frequency is $2.5 \mathrm{GHz}$. The channel code used is the rate- $(1 / 2)$ convolutional code with generator polynomial ([171, 133]) with random interleaving. To study the performance of different channel estimation schemes, we use the following frame structure with four OFDM symbols, one preamble and one postamble. In our proposed channel estimation scheme A, the pilot-density is (1/7) sub-carriers, which is the same as that of the Partial Usage of Sub-Carriers (PUSC) mode of the WiMAX standard, but their locations are based on the pilot pattern given in Fig. 5. In our proposed channel estimation scheme B, only the gray pilots in Fig. 5 are used.

The channel's power delay profile is that of SUI-3 specification with mobility according to the Jakes model and a normalized Doppler spread of 5\% corresponding to a speed of 235 $\mathrm{km} / \mathrm{h}$. The I/Q imbalance parameters are $\theta_{t}=\theta_{r}=5^{\circ}$ and $\alpha_{t}=\alpha_{r}=0.5 \mathrm{~dB}$. For the case of the FD I/Q imbalance, we adopt the simulation settings from [5] with $\bar{\xi}_{t}^{I}(t)=\bar{\xi}_{r}^{I}(t)=$ $0.01 \delta(t)+\delta(t-T)+0.01 \delta(t-2 T)$ and $\bar{\xi}_{t}^{Q}(t)=\bar{\xi}_{r}^{Q}(t)=$ $0.01 \delta(t)+\delta(t-T)+0.2 \delta(t-2 T)$. The FIR MMSE-FEQ in Section $\mathrm{V}$ generates soft data estimates which are fed to the Viterbi convolutional decoder.

\section{B. Results and Discussion}

Figs. 6-8 show the BER performances for 4, 16, and 64-QAM constellations, respectively. Results have been generated both for uncompensated and compensated scenarios, as discussed in Section IV, for $Q=3$ and 5. We see considerable performance improvements for $Q=3$ compared to the uncompensated case. Furthermore, the performance gains in going from $Q=3$ to 5 is significant for large signal constellations, as they are more sensitive to ICI. Fig. 7 demonstrates that joint compensation for I/Q imbalance and mobility results in significant performance gain compared to separate compensation I/Q imbalance or mobility only.

Fig. 9 compares channel estimation schemes A and B under FI I/Q imbalance with 16-QAM. Scheme-B achieves almost the same performance as Scheme-A and both perform within a $2 \mathrm{~dB}$ $\left(E_{b} / N_{0}\right)$ loss compared to the case of perfect knowledge of channel state information (CSI) and I/Q imbalance parameters. Fig. 10 compares the performance for 16-QAM with FI and FD I/Q imbalances. It is clear that our proposed channel estimation and compensation schemes are quite effective in mitigating FD I/Q imbalance even at high mobility.

\section{CONCLUSION}

In this paper, we developed a generalized input-output model for mobile OFDM systems under both FI and FD joint transmit/ receive I/Q imbalances. Based on this generalized model, we derived a closed-form SINR expression as a function of the I/Q imbalance parameters and Doppler spread. In addition, we presented a reduced-complexity FIR MMSE-FEQ digital baseband compensation scheme and proposed an efficient channel estimation scheme which can be used for both FI and FD I/Q imbalances. To reduce the pilot overhead, we proposed a second channel estimation scheme for FI I/Q imbalance. Our simulation results show that our proposed joint compensation scheme using six taps per sub-carrier achieves significant performance gains compared to the uncompensated case and also to schemes that

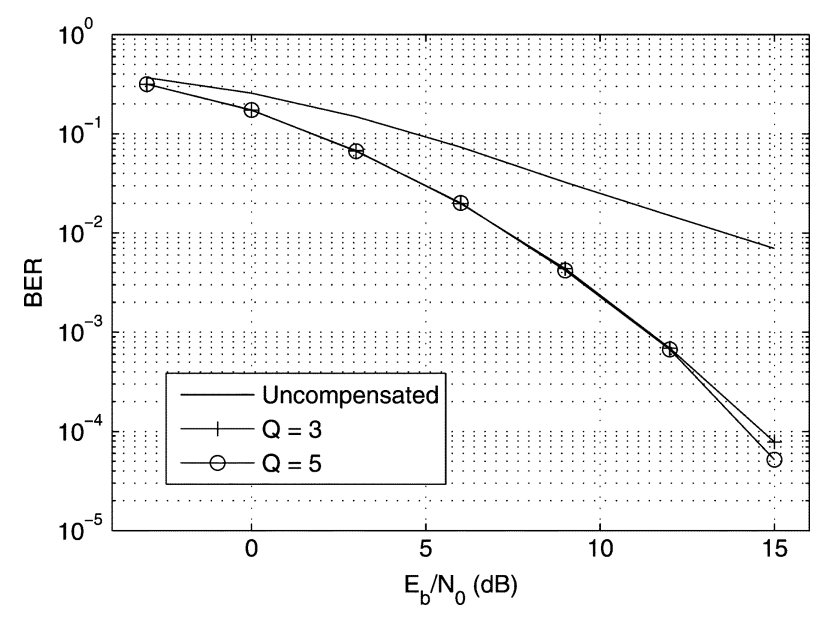

Fig. 6. BER Performance for 4-QAM constellation with frequency-independent I/Q imbalance, Perfect CSI.

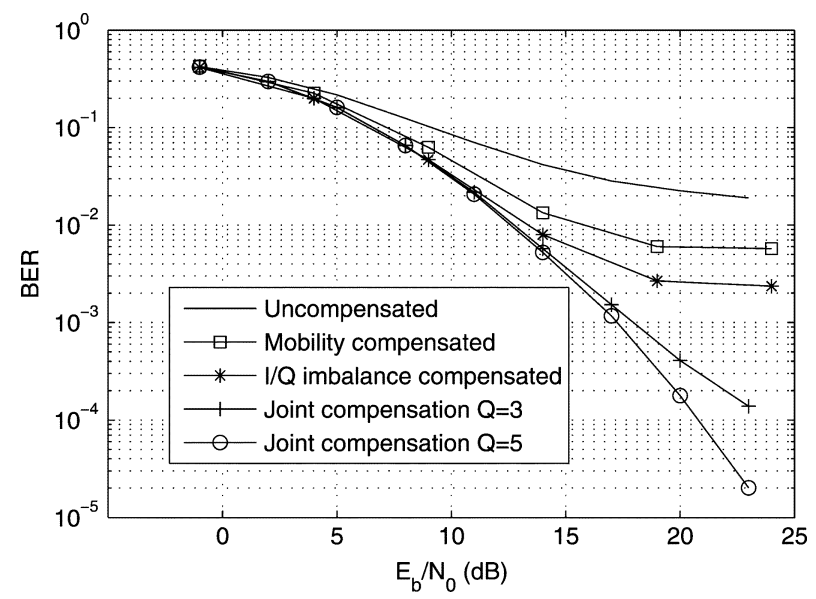

Fig. 7. BER Performance for 16-QAM constellation with FI I/Q imbalance, Perfect CSI.

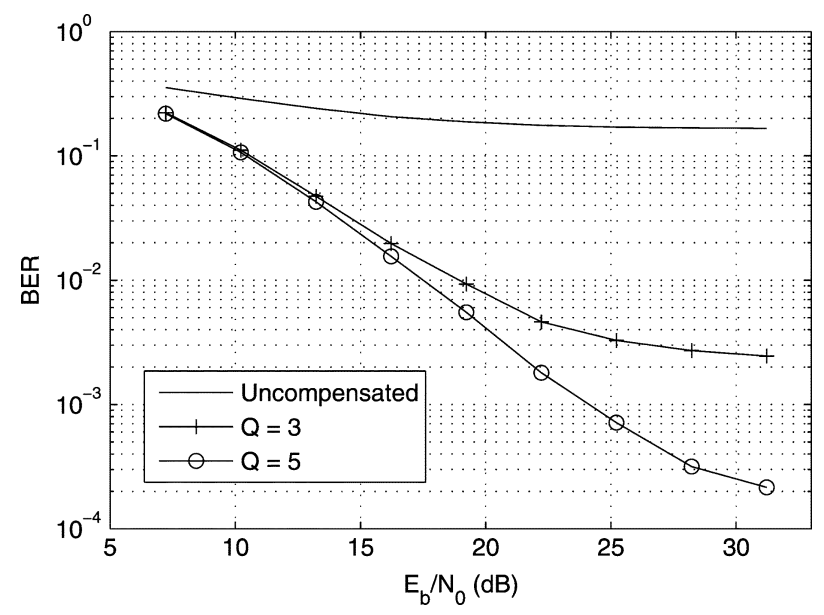

Fig. 8. BER Performance for 64-QAM constellation with FI I/Q imbalance, Perfect CSI.

compensate for I/Q imbalance only or mobility only. The BER results of our proposed schemes are only within $2 \mathrm{~dB}\left(E_{b} / N_{0}\right)$ loss from an ideal system with perfect knowledge of CSI and I/Q imbalance parameters. 


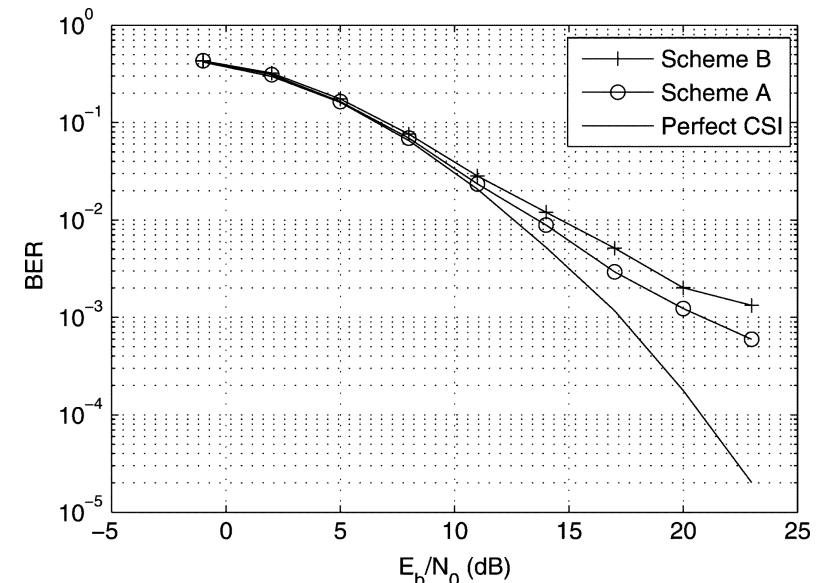

Fig. 9. BER comparison of channel estimation Schemes A and B for 16-QAM constellation under FI I/Q imbalance, $Q=5$.

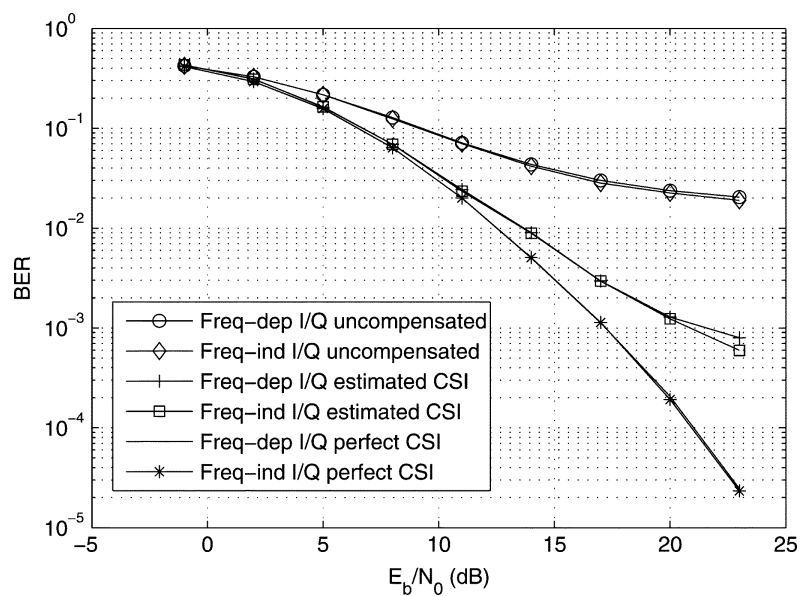

Fig. 10. BER comparison of channel estimation schemes with perfect CSI for 16-QAM with FD and FI I/Q imbalances.

\section{APPENDIX A}

\section{SINR DEGRADATION ANALYSIS}

In the section, all indices are from 0 to $N-1$ and will be suppressed for brevity. Moreover, multiple summations have also been combined together for brevity. For SINR analysis, we start by proving the following.

Proposition: Given the Assumption-1 in Section III, it is true that

$$
\begin{aligned}
& E\left\{g\left(k_{1}, l_{1}\right) g\left(k_{2}, l_{2}\right)\right\}=0 \\
& E\left\{|g(k, l)|^{2}\right\} \\
& \quad=\frac{1}{N^{2}} \sum_{n_{1}, n_{2}} R\left(n_{1}, n_{2}\right) e^{-j \frac{2 \pi}{N}(k-l)\left(n_{1}-n_{2}\right)}
\end{aligned}
$$

where $R\left(n_{1}, n_{2}\right)$ is the normalized time-correlation function which is the same for each tap and the relation between $g(l, k)$ and $\bar{g}(n, m)$ is given by (24).
Proof: From the assumption that $\bar{g}(n, m)$ is circularly symmetric, we have the mean $E\{\bar{g}(n, m)\}=0$. Combined with the fact that taps are uncorrelated for different $m$, this would imply that

$$
E\left\{\bar{g}\left(n_{1}, m_{1}\right) \bar{g}\left(n_{2}, m_{2}\right)\right\}= \begin{cases}R_{m}^{p}\left(n_{1}, n_{2}\right), & m_{1}=m_{2}=m \\ 0, & m_{1} \neq m_{2} .\end{cases}
$$

Moreover, $R_{m}^{p}\left(n_{1}, n_{2}, m\right)=E\left\{\bar{g}\left(n_{1}, m\right) \bar{g}\left(n_{2}, m\right)\right\}$ is the pseudo-covariance function for tap $m$. Because of the circular-symmetry assumption, this is equal to zero. From the assumption of uncorrelated taps, we have

$$
E\left\{\bar{g}\left(n_{1}, m_{1}\right) \bar{g}^{*}\left(n_{2}, m_{2}\right)\right\}= \begin{cases}R_{m}\left(n_{1}, n_{2}\right), & m_{1}=m_{2}=m \\ 0, & m_{1} \neq m_{2}\end{cases}
$$

where $\left.R_{m}\left(n_{1}, n_{2}\right)=E\left\{\bar{g}\left(n_{1}, m\right) \bar{g}^{*}\left(n_{2}, m\right)\right)\right\}$ is the covariance function of tap $m$, but each tap has the same statistical behavior differing only in their average powers. Therefore, we can write $R_{m}\left(n_{1}, n_{2}\right)=\sigma_{\bar{g}_{m}}^{2} R\left(n_{1}, n_{2}\right)$, where $\sigma_{\bar{g}_{m}}^{2}$ is the average power of tap $m$ and $R\left(n_{1}, n_{2}\right)$ is the normalized covariance function common to all taps. So, we have the following:

$$
\begin{aligned}
& E\left\{\bar{g}\left(n_{1}, m_{1}\right) \bar{g}\left(n_{2}, m_{2}\right)\right\}=0 \\
& E\left\{\bar{g}\left(n_{1}, m_{1}\right) \bar{g}^{*}\left(n_{2}, m_{2}\right)\right\}=\sigma_{\bar{g}_{m_{1}}}^{2} R\left(n_{1}, n_{2}\right) \delta\left(m_{1}-m_{2}\right) .
\end{aligned}
$$

Using (24) and (47), we can obtain (49), where $Q 1=k_{1} n_{1}-$ $\left(n_{1}-m_{1}\right) l_{1}$ and $Q_{2}=k_{2} n_{2}-\left(n_{2}-m_{2}\right) l_{2}$. Moreover, using (24) and (48), we have (50), where $m_{1}=m_{2}=m$. For the special case of $k_{1}=k_{2}$ and $l_{1}=l_{2}$ and utilizing Assumption-III of Section III, the above equation simplifies to (46). This completes the proof of the proposition. Equation (46) suggests that the average power of the $(k, l)$ element in the frequency-domain channel matrix is dependent only on the difference $(k-l)$, i.e., elements in the same diagonal have the same average power.

$$
\begin{aligned}
& E\left\{g\left(k_{1}, l_{1}\right) g\left(k_{2}, l_{2}\right)\right\} \\
& =\frac{1}{N^{2}} \sum_{m_{1}, n_{1}, m_{2}, n_{2}} E\left\{\bar{g}\left(n_{1}, m_{1}\right) \bar{g}\left(n_{2}, m_{2}\right)\right\} \\
& \quad \times e^{-j \frac{2 \pi}{N}\left(Q_{1}+Q_{2}\right)}=0 \\
& E\left\{g\left(k_{1}, l_{1}\right) g^{*}\left(k_{2}, l_{2}\right)\right\} \\
& =\frac{1}{N^{2}} \sum_{m_{1}, n_{1}, m_{2}, n_{2}} E\left\{\bar{g}\left(n_{1}, m_{1}\right) \bar{g}^{*}\left(n_{2}, m_{2}\right)\right\} \\
& \quad \times e^{-j \frac{2 \pi}{N}\left(Q_{1}-Q_{2}\right)} \\
& =\frac{1}{N^{2}} \sum_{m} \sigma_{\bar{g}_{m}}^{2} e^{-j \frac{2 \pi}{N} m\left(l_{1}-l_{2}\right)} \\
& \quad \times \sum_{n_{1}, n_{2}} R\left(n_{1}, n_{2}\right) e^{-j \frac{2 \pi}{N}\left(\left(k_{1} n_{1}-k_{2} n_{2}\right)-\left(l_{1} n_{1}-l_{2} n_{2}\right)\right)}
\end{aligned}
$$


Next, we calculate the energy of $S(k)$ and $I(k)$ using the proposition. From (26), we know that

$S(k)$

$$
\begin{aligned}
= & \underbrace{\left[\lambda_{r}(k) \lambda_{t}(k) g(k, k)+\phi_{r}(k) \phi_{t}^{*}\left(k^{\prime}\right) g^{*}\left(k^{\prime}, k^{\prime}\right)\right]}_{a} s(k) \\
& +\underbrace{\left[\lambda_{r}(k) \phi_{t}\left(k^{\prime}\right) g\left(k, k^{\prime}\right)+\phi_{r}(k) \lambda_{t}^{*}(k) g^{*}\left(k^{\prime}, k\right)\right]}_{b} s^{*}(k) .
\end{aligned}
$$

$\Rightarrow E\left\{|S(k)|^{2}\right\}=E\left\{|s(k)|^{2}\left(|a|^{2}+|b|^{2}\right)+2 \Re\left\{a b^{*} s^{2}(k)\right\}\right\}$.

Let $a b^{*}=C_{R}+j C_{I}$ and $s(k)=s_{R}+j s_{I}$. Then, (52) is true. As a result of Assumption-2 of Section III, we have $E\left\{s_{R}^{2}\right\}=E\left\{s_{I}^{2}\right\}$ and $E\left\{s_{R}\right\}=E\left\{s_{I}\right\}=0$, and hence $E\left\{\Re\left(a b^{*} s^{2}(k)\right)\right\}=0$. Then, $E\left\{|S(k)|^{2}\right\}$ is given by (53)

$$
\begin{aligned}
& E\left\{\Re\left\{a b^{*} s^{2}(k)\right\}\right\} \\
&= E\left\{C_{R}\left(s_{R}^{2}-s_{I}^{2}\right)-2 C_{I} s_{R} s_{I}\right\} \\
&= E\left\{C_{R}\right\} E\left\{s_{R}^{2}-s_{I}^{2}\right\} \\
&-2 E\left\{C_{I}\right\} E\left\{s_{R}\right\} E\left\{s_{I}\right\} \\
& E\left\{|S(k)|^{2}\right\} \\
&= \frac{1}{N^{2}} E_{s}\left[\left|\lambda_{r}(k)\right|^{2}\left|\lambda_{t}(k)\right|^{2} \sum_{n_{1}, n_{2}} R\left(n_{1}, n_{2}\right)\right. \\
&+\left|\phi_{r}(k)\right|^{2}\left|\phi_{t}\left(k^{\prime}\right)\right|^{2} \sum_{n_{1}, n_{2}} R\left(n_{1}, n_{2}\right) \\
&+\left|\lambda_{r}(k)\right|^{2}\left|\phi_{t}\left(k^{\prime}\right)\right|^{2} \\
& \times \sum_{n_{1}, n_{2}} R\left(n_{1}, n_{2}\right) e^{-j \frac{2 \pi}{N}\left(2 k\left(n_{1}-n_{2}\right)\right)} \\
&+\left|\phi_{r}(k)\right|^{2}\left|\lambda_{t}(k)\right|^{2} \\
&\left.\times \sum_{n_{1}, n_{2}} R\left(n_{1}, n_{2}\right) e^{j \frac{2 \pi}{N}\left(2 k\left(n_{1}-n_{2}\right)\right)}\right]
\end{aligned}
$$

where we have used the proposition to compute $E\left\{|g(\cdot, \cdot)|^{2}\right\}$

Now, we need to compute the energy of the interference term $I(k)$. The interference term in (28) can be rewritten as

$$
\begin{aligned}
I(k)= & \sum_{l \neq k}(\underbrace{\lambda_{r}(k) \lambda_{t}(l) g(k, l) s(l)}_{I_{1}(l)}+\underbrace{\phi_{r}(k) \phi_{t}^{*}\left(l^{\prime}\right) g^{*}\left(k^{\prime}, l^{\prime}\right) s(l)}_{I_{2}(l)} \\
& +\underbrace{\lambda_{r}(k) \phi_{t}\left(l^{\prime}\right) g\left(k, l^{\prime}\right) s^{*}(l)}_{I_{3}(l)}+\underbrace{\phi_{r}(k) \lambda_{t}^{*}(l) g^{*}\left(k^{\prime}, l\right) s^{*}(l)}_{I_{4}(l)}) .
\end{aligned}
$$

We have introduced the new symbols $I_{1}(l), I_{2}(l), I_{3}(l)$ and $I_{4}(l)$, so we can write (54), where all terms of the type $E\left\{I_{p}\left(l_{1}\right) I_{q}^{*}\left(l_{2}\right)\right\}$ with $p \neq q$ and of the type $E\left\{I_{p}\left(l_{1}\right) I_{p}^{*}\left(l_{2}\right)\right\}$ with $l_{1} \not l_{2}$ are zero, either because of (45) or because $E\left\{s\left(l_{1}\right) s\left(l_{2}\right)\right\}=0$ which is a consequence of Assumption-2 of Section III. Using (46) and noting that $R\left(n_{1}, n_{2}\right)$ is the normalized time-correlation function, we have (55) to (58), and $E\left\{|I(k)|^{2}\right\}=E I_{1}+E I_{2}+E I_{3}+E I_{4}$.

$$
\begin{aligned}
E\left\{|I(k)|^{2}\right\}= & \sum_{l_{1}, l_{2} \neq k} E\left\{\left[I_{1}\left(l_{1}\right)+I_{2}\left(l_{1}\right)\right.\right. \\
& \left.+I_{3}\left(l_{1}\right)+I_{4}\left(l_{1}\right)\right]\left[I_{1}^{*}\left(l_{2}\right)+I_{2}^{*}\left(l_{2}\right)\right. \\
& \left.\left.+I_{3}^{*}\left(l_{2}\right)+I_{4}^{*}\left(l_{2}\right)\right]\right\} . \\
E I_{1}= & \sum_{l} E\left\{\left|I_{1}(l)\right|^{2}\right\}-E\left\{\left|I_{1}(k)\right|^{2}\right\} \\
= & \frac{E_{s}}{N^{2}}\left|\lambda_{r}(k)\right|^{2}\left(\sum_{n_{1}, n_{2}, l} R\left(n_{1}, n_{2}\right)\left|\lambda_{t}(l)\right|^{2}\right. \\
& \times e^{-j \frac{2 \pi}{N}(k-l)\left(n_{1}-n_{2}\right)} \\
& \left.-\left|\lambda_{t}(k)\right|^{2} \sum_{n_{1}, n_{2}} R\left(n_{1}, n_{2}\right)\right) \\
E I_{2}= & \frac{E_{s}}{N^{2}}\left|\phi_{r}(k)\right|^{2}\left(\sum_{n_{1}, n_{2}, l} R\left(n_{1}, n_{2}\right)\left|\phi_{t}\left(l^{\prime}\right)\right|^{2}\right. \\
& \times e^{-j \frac{2 \pi}{N}\left(k^{\prime}-l^{\prime}\right)\left(n_{1}-n_{2}\right)} \\
& \left.-\left|\phi_{t}\left(k^{\prime}\right)\right|^{2} \sum_{n_{1}, n_{2}} R\left(n_{1}, n_{2}\right)\right) \\
& \left.-\left|\lambda_{t}(k)\right|^{2} \sum_{n_{1}, n_{2}} R\left(n_{1}, n_{2}\right)\right) . \\
E I_{3}= & \frac{E_{s}}{N^{2}}\left|\lambda_{r}(k)\right|^{2}\left(\sum_{n_{1}, n_{2}, l} R\left(n_{1}, n_{2}\right)\left|\phi_{t}\left(l^{\prime}\right)\right|^{2}\right. \\
& \times e^{-j \frac{2 \pi}{N}\left(k-l^{\prime}\right)\left(n_{1}-n_{2}\right)} \\
& \left.-\left|\phi_{t}\left(k^{\prime}\right)\right|^{2} \sum_{n_{1}, n_{2}} R\left(n_{1}, n_{2}\right)\right) \\
E I_{4}= & \frac{E_{s}}{N^{2}}\left|\phi_{r}(k)\right|^{2}\left(\sum_{n_{1}, n_{2}, l} R\left(n_{1}, n_{2}\right)\left|\lambda_{t}(l)\right|^{2}\right. \\
& \times e^{-j \frac{2 \pi}{N}\left(k^{\prime}-l\right)\left(n_{1}-n_{2}\right)} \\
&
\end{aligned}
$$

Colored Noise Variance: Consider the noise term in (15), namely $\mathbf{v}=\mathbf{F} \overline{\mathbf{v}}=\mathbf{F} \overline{\mathbf{\Lambda}}_{\mathbf{r}}^{\prime} \overline{\mathbf{w}}+\mathbf{F} \overline{\mathbf{\Phi}}_{\mathbf{r}}^{\prime} \overline{\mathbf{w}}^{*}$. The vector $\mathbf{v}$ is Gaussian distributed with zero-mean and its covariance matrix is given by

$$
E\left\{\mathbf{v} \mathbf{v}^{H}\right\}=\sigma^{2}\left(\mathbf{F} \overline{\boldsymbol{\Lambda}}_{\mathbf{r}}^{\prime} \overline{\boldsymbol{\Lambda}}_{r}^{\prime}{ }^{H} \mathbf{F}^{H}+\mathbf{F} \overline{\mathbf{\Phi}}_{\mathbf{r}}^{\prime} \overline{\mathbf{\Phi}}_{\mathbf{r}}^{\prime H} \mathbf{F}^{H}\right)
$$

where $E\{\overline{\mathbf{w}}\}=0, E\left\{\overline{\mathbf{w}} \overline{\mathbf{w}}^{T}\right\}=\mathbf{0}_{\left(N+N_{\mathrm{CP}}\right) \times\left(N+N_{\mathrm{CP}}\right)}$, $E\left\{\overline{\mathbf{w}} \overline{\mathbf{w}}^{H}\right\}=\sigma^{2} \mathbf{I}_{\left(N+N_{\mathrm{CP}}\right)}$. To get an expression for the noise power on the $k$ th sub-carrier, define $\zeta=$ $\operatorname{Diag}\left(\mathbf{F} \overline{\boldsymbol{\Lambda}}_{\mathbf{r}}^{\prime} \overline{\boldsymbol{\Lambda}}_{\mathbf{r}}^{\prime H} \mathbf{F}^{H}+\mathbf{F} \overline{\boldsymbol{\Phi}}_{\mathbf{r}}^{\prime} \overline{\mathbf{\Phi}}_{\mathbf{r}}^{\prime H} \mathbf{F}^{H}\right)$. If $\zeta(k)$ is the $k$ th element of $\zeta$, then

$$
E\left\{|v(k)|^{2}\right\}=\sigma^{2} \zeta(k) .
$$




\section{REFERENCES}

[1] C. L. Liu, "Impacts of I/Q imbalance on QPSK-OFDM-QAM detection," IEEE Trans. Consum. Electron., vol. 44, no. 3, pp. 984-989, Aug. 1998.

[2] A. Schuchert, R. Hasholzne, and P. Anotine, "A novel I/Q imbalance compensation scheme for the reception of OFDM signals," IEEE Trans. Consum. Electron., vol. 47, no. 3, pp. 313-318, 2001.

[3] A. Tarighat, R. Bagheri, and A. H. Sayed, "Compensation schemes and performance analysis of IQ imbalances in OFDM receivers," IEEE Trans. Signal Process., vol. 53, no. 8, pp. 3257-3268, Aug. 2005.

[4] A. Tarighat and A. H. Sayed, "Joint compensation of transmitter and receiver impairments in OFDM systems," IEEE Trans. Wireless Commun., vol. 6, no. 1, pp. 240-247, Jan. 2007.

[5] M. Valkama, M. Renfors, and V. Koivunen, "Compensation of frequency-selective I/Q imbalances in wideband receivers: Models and algorithms," in Proc. IEEE 3rd Workshop Signal Process. Adv. in Wireless Commun. (SPAWC'01), Mar. 2001, pp. 42-45.

[6] J. Tubbax, B. Come, L. V. der Perre, L. Deneire, S. Donnay, and M. Engels, "Compensation of IQ imbalance in OFDM systems," in Proc. IEEE ICC, May 2003, vol. 5, pp. 3403-3407.

[7] P. Rykaczewski, J. Brakensiek, and F. Jondral, "Decision directed methods of I/Q imbalance compensation in OFDM systems," in Proc. IEEE VTC, Sep. 2004, vol. 1, pp. 484-487.

[8] D. Tandur and M. Moonen, "Joint adaptive compensation of transmitter and receiver IQ imbalance under carrier frequency offset in OFDM-based systems," IEEE Trans. Signal Process., vol. 55, no. 11, pp. 5246-5252, Nov. 2007.

[9] S. Lu, R. Kalbasi, and N. Al-Dhahir, "OFDM interference mitigation algorithms for doubly-selective channels," in IEEE Veh. Technol. Conf., Sep. 2006, pp. 1-5.

[10] G. Stuber, Principles of Mobile Communication.. New York: Springer, 2000.

[11] B. Narasimhan, S. Lu, N. Al-Dhahir, and H. Minn, "Digital baseband compensation of I/Q imbalance in mobile OFDM," in Proc. IEEE Wireless Commun. Netw. Conf., Las Vegas, NV, Mar. 2008, pp. 646-651.

[12] C. F. V. L. G. H. Golub, Matrix Computations.. Baltimore, MD: Johns Hopkins Univ. Press, 1996.

[13] S. Kay, Fundamentals of Statistical Signal Processing: Estimation Theory.. Englewood Cliffs, NJ: Prentice-Hall, 1993.

[14] WiMAX standard, IEEE Std 802.16e-2005 and IEEE Std 802.16-2004/ Corl-2005, Feb. 28, 2006.

[15] Y. Mostofi and D. C. Cox, "ICI mitigation for pilot-aided OFDM mobile systems," IEEE Trans. Wireless Commun., vol. 4, no. 3, pp. 765-774, Mar. 2005.

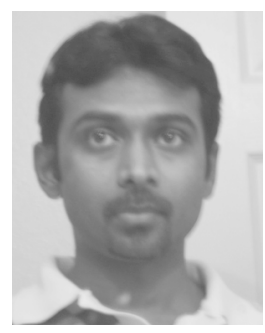

Balachander Narasimhan (S'07) received the B.E. degree in electronics and communication engineering from University of Madras, Chennai, India, in 2000 and the M.Tech. degree from the Indian Institute of Technology Bombay, Mumbai, India, in 2003. He is currently pursuing the $\mathrm{Ph} . \mathrm{D}$. degree at the University of Texas at Dallas, Richardson.

He worked for HP Labs India from 2003-2004 and Midas Communication Technologies Pvt., Ltd., from 2004 to 2006 as Design Lead working on WiMax simulation studies. His research focuses on signal processing and equalization techniques to mitigate RF impairments in the baseband.

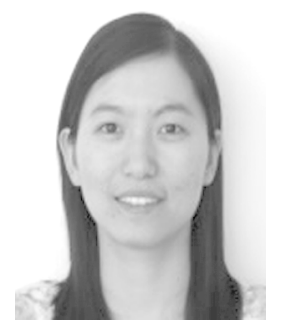

Dandan Wang (S'05) received the B.S. and M.S. degrees in electrical engineering from Beijing University of Posts and Telecommunications, Beijing, China, in 2000 and 2003, respectively, and the $\mathrm{Ph} . \mathrm{D}$. degree from the Department of Electrical and Computer Engineering, University of Texas at Dallas, Richardson, in 2008

She worked at the China Radio Research Lab, Ericsson, from 2003 to 2004. From August, 2004 to August, 2005, she was with Department of Electrical and Computer Engineering, Stevens Institute of Technology, Hoboken, NJ. She is now with Huawei Technologies. Her research interests include wireless communications and networking, signal processing for communications and sensor networks, cognitive radio, and cooperative/relay systems.

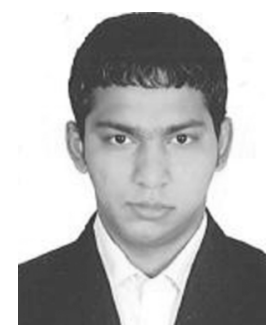

Systems Group.

Mr. Narayanan is a recipient of Jonsson Distinguished Scholarship at UTD, during the course of his study.

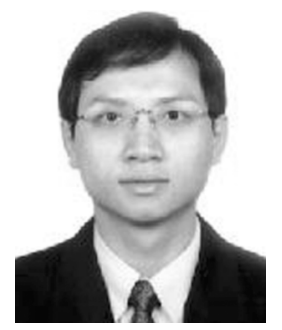

Hlaing Minn (S'99-M'01-SM'07) received the B.E. degree in electronics from the Yangon Institute of Technology, Yangon, Myanmar, in 1995, the M.Eng. degree in telecommunications from the Asian Institute of Technology (AIT), Pathumthani, Thailand, in 1997, and the Ph.D. degree in electrical engineering from the University of Victoria, Victoria, BC, Canada, in 2001

He was with the Telecommunications Program in AIT as a Laboratory Supervisor during 1998. He was a Research Assistant from 1999 to 2001 and a Postdoctoral Research Fellow during 2002 in the Department of Electrical and Computer Engineering at the University of Victoria. He has been with the Erik Jonsson School of Engineering and Computer Science, the University of Texas at Dallas, Richardson, since 2002, and currently is an Associate Professor. His research interests include wireless communications, statistical signal processing, error control, detection, estimation, synchronization, signal design, cross-layer design, cognitive radios, and wireless health-care applications.

Prof. Minn is an Editor for the IEEE TRANSACTIONS ON COMMUNICATIONS and International Journal of Communications and Networks.

Naofal Al-Dhahir (F'08) for biography and photo see the Editorial of this special issue. 